\title{
Palmitoyl Acyltransferase zD17 Mediates Neuronal Responses in Acute Ischemic Brain Injury by Regulating JNK Activation in a Signaling Module
}

\author{
Guang Yang and Max S. Cynader \\ Brain Research Centre, Vancouver, British Columbia, Canada, V6T 2B5
}

\begin{abstract}
Although the palmitoyl acyltransferase (PAT) zinc-finger DHHC containing 17 (zD17) has been implicated in genetic neurological disorders by regulating protein palmitoylation, the role of zD17 in acute brain injury remains unknown. Here, we report that zD17 contributes to acute ischemic brain injury via a mechanism independent of its PAT activity. We have found that zD17 directly interacts with c-Jun $\mathrm{N}$ terminus kinase (JNK) to form a signaling module for JNK activation. Pathological stressors induce the zD17-JNK interaction, which promotes downstream neuronal cell death signals. We have developed novel peptides targeting the JNK-interacting motif on zD17 to selectively block the enhancement of the zD17-JNK interaction and the activation of JNK isoforms 2 and 3. Application of these peptides successfully blocks JNK activation and neuronal cell death pathways, protects cultured neurons from excitotoxicity, and dramatically reduces brain damage and behavioral deficits in a rat model of focal ischemic stroke. Our findings indicate zD17 as a key player in ischemic stroke and suggest the potential therapeutic value of targeting the zD17-JNK interaction for acute brain injury.
\end{abstract}

\section{Introduction}

Stroke is a leading cause of death and disability worldwide (WHO, 2007). In acute ischemic stroke, the most common form of stroke, the supply of blood and oxygen to a part of the brain is reduced, which eventually causes the death of brain tissue and forms the infarct core (Donnan et al., 2008). This irreversibly damaged core is surrounded by a dysfunctional but potentially viable hypoperfused region, known as the penumbra. Within a few hours after ischemia, neurons in the penumbra are challenged by excitotoxic and inflammatory processes resulting in delayed death (Fisher and Ratan, 2003; Bossy-Wetzel et al., 2004).

Zinc-finger DHHC containing 17 (zD17) belongs to a family of palmitoyl acyltransferases (PATs) that catalyze protein palmitoylation, a posttranslational lipid modification affecting protein trafficking and function (el-Husseini and Bredt, 2002; Singaraja et al., 2002; Huang et al., 2004). Through its regulation of the poly-Q-repeat mutated huntingtin ( $\mathrm{Htt}$ ) protein's palmitoylation and aggregation, zD17 has been implicated in neurodegeneration in Huntington's disease (Yanai et al., 2006). However, whether zD17 is also involved in neuronal cell death in the ab-

Received May 19, 2011; revised June 21, 2011; accepted June 23, 2011.

Author contributions: G.Y. and M.S.C. designed research; G.Y. performed research; G.Y. and M.S.C. contributed unpublished reagents/analytic tools; G.Y. and M.S.C. analyzed data; G.Y. and M.S.C. wrote the paper.

This work was supported by grants from the Canadian Institutes of Health Research (M.S.C.) and Natural Sciences and Engineering Research Council of Canada (M.S.C.). We thank Dr. Vsevolod V. Gurevich for providing the GFP-JNK3 construct. We also thank Dr. Rujun Kang from Dr. Alaa Husseini's laboratory for providing DNA constructs. We are grateful to Wendy Wen for preparing neuron cultures and assisting in double-blinded animal studies. We thank Ted Lai and Jing Wang for their technical assistance and Drs. YuTian Wang and Luba Kojic for critical reading of this manuscript. We also thank the members of the laboratory of M.S.C. for helpful suggestions.

Correspondence should be addressed to either Guang Yang or Max S. Cyander, Brain Research Centre, 2211 Wesbrook Mall, Vancouver, BC, Canada V6T 2B5. E-mail: photonyg@interchange.ubc.ca or cynader@brain.ubc.ca. DOI:10.1523/JNEUROSCI.2510-11.2011

Copyright $\odot 2011$ the authors $\quad 0270-6474 / 11 / 3111980-12 \$ 15.00 / 0$ sence of mutated Htt protein is not known. In addition to acting as a PAT, zD17 seems to have other roles (Goytain et al., 2008), including the activation of c-Jun $\mathrm{N}$ terminus kinase (JNK) pathways (Harada et al., 2003). JNK activation is broadly involved in excitotoxicity, inflammation, and neuronal cell death in ischemic stroke as well as other neurological diseases (Resnick and Fennell, 2004; Weston and Davis, 2007). It is thus possible that $\mathrm{zD} 17$ may contribute to acute neuronal cell death, for example in ischemic stroke, via JNK pathways, rather than via controlling Htt aggregation. In our pilot studies leading to this report, we found that zD17-induced JNK activation is independent of its PAT activity. We thus investigate this PAT-independent role of zD17 in regulating ischemic stroke-induced acute neuronal cell death.

JNK initiates both immediate and late-phase cell death events via phosphorylation and activation of substrates in both the nucleus and cytosol (Bredesen et al., 2006; Centeno et al., 2007; Weston and Davis, 2007). Inhibitors targeting JNK have been shown promising neuroprotection in neurological diseases, despite concerns of side effects raised in clinical trials (Kuan and Burke, 2005; Bogoyevitch, 2006; Borsello and Forloni, 2007). Studies on the three JNK isoforms have pinpointed different roles of JNK1, JNK2, and JNK3 in neuronal functions and neurological diseases. Although $J N K 2^{-/-}, J N K 3^{-/-}$or $J N K 2^{-/-}, J N K 3^{-/-}$ mice show reduced vulnerability in models of ischemic stroke, $J N \mathrm{~K}^{-/-}$animals show little protective effect (Hunot et al., 2004). Moreover, the loss of JNK1 leads to disorganization of neuronal microtubules and neurodegeneration (Chang et al., 2003). These results strongly suggest that selective inhibition of stress-responsive JNK isoforms (primarily JNK2/3) would be beneficial for therapeutic targeting of JNK (Coffey et al., 2002; Bogoyevitch, 2006). However, the design of JNK inhibitors with clear isoform selectivity and the therapeutic application of these 
A

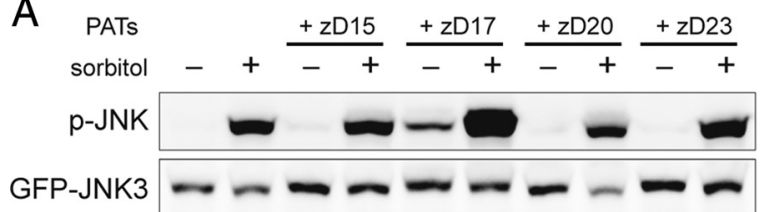

B

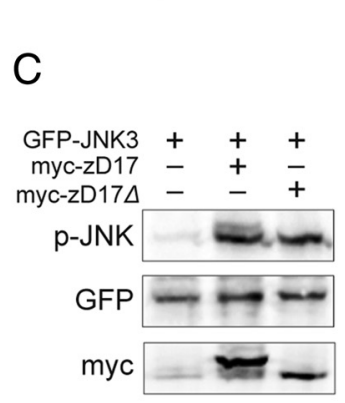

$\mathrm{D}$

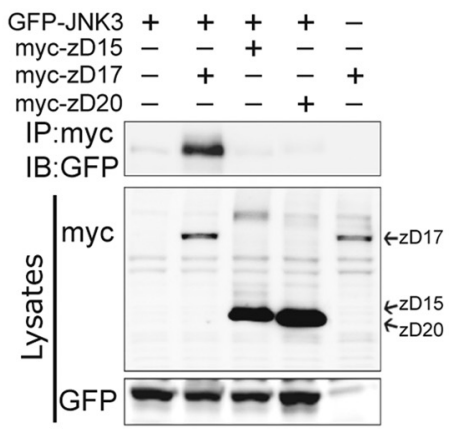

$\mathrm{E}$
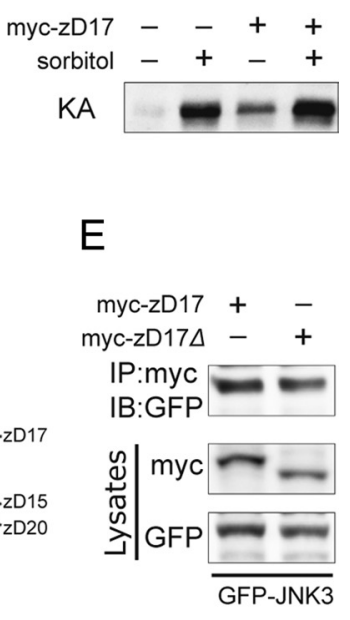

$\mathrm{F}$

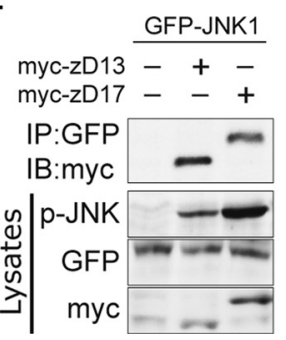

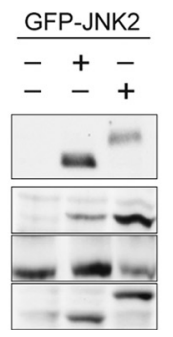

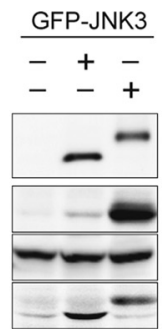

G

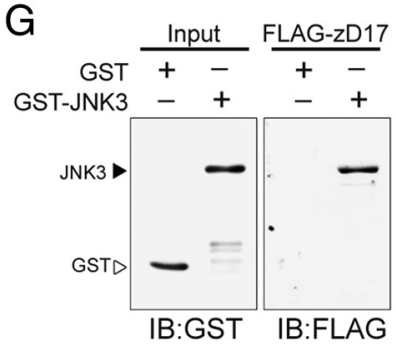

Figure 1. Identification of ZD17 as a JNK-interacting protein. $A$, ZD17 promotes JNK3 phosphorylation. Under both resting and osmotic stress (400 mm sorbitol, $30 \mathrm{~min}$ ) conditions, the phosphorylation level of GFP-JNK3 in heterogeneous HEK293 cells is enhanced by coexpression of myc-ZD17 but not PAT myc-ZD15, ZD20, or zD23. B, JNK3 activity is promoted by zD17, as assessed by kinase assays (KA) (fold change compared with JNK3 alone; JNK3 + zD17, $4.8 \pm 0.2, p<0.01$; sorbitol, $12.4 \pm 0.2$; JNK3 + zD17 + sorbitol, $15.3 \pm 0.6, p<0.01$ compared with sorbitol alone). $t$ test. Quantifications show means \pm SEM. C, ZD17 promotes JNK3 phosphorylation in a PAT activity-independent manner. JNK3 phosphorylation is enhanced by both wild-type ZD17 (fold change to JNK3 only; $9.98 \pm 1.00 ; p<0.01$ ) and the PAT-activity-deficient mutant ZD17 $\Delta(10.61 \pm 1.09 ; p<0.01) . \boldsymbol{D}$, JNK3 associates with ZD17 in HEK293 cells. GFP-JNK3 is present in the ZD17 immunoprecipitates enriched by anti-myc antibody but is not detected in ZD15 or ZD20 immunoprecipitates. $E$, ZD17 associates with JNK3 independent of its PAT activity. Both ZD17 wild-type and zD17 $\Delta$ are capable of interacting with JNK3, as assessed by coimmunoprecipitation. $\boldsymbol{F}, \mathrm{zD} 17$ interacts with JNK1 and JNK2 and promotes their phosphorylation. The heterogeneously expressed PAT ZD13 interacts with JNKs but fails to promote JNK phosphorylation. G, Purified GST-JNK3 physically binds to myc-ZD17 in vitro. IP, Immunoprecipitation; IB, immunoblot.

inhibitors in ischemic stroke have not been achieved. Here, we report a role of zD17 in mediating ischemic neuronal death by recruiting JNK pathways and develop a neuroprotective JNK inhibitor with the isoform and scenario selectivity.

\section{Materials and Methods}

Cell cultures and materials. All animal experiments were approved by the Animal Care Committee of the University of British Columbia. Primary cortical or hippocampal neuronal cultures were prepared from Wistar rat (University of British Columbia Animal Care Centre, Vancouver, BC, Canada) brains at embryonic days $18-19$. Cultures were maintained in Neurobasal medium supplemented with B27 and $0.5 \mathrm{~mm}$ glutamax (Invitrogen) as described previously (Yang et al., 2009). For biochemical studies, cortical neurons were seeded in six-well culture plates with a density of $7.5 \times 10^{5}$ cells per well. For lactate dehydrogenase (LDH) assays and cell death detection, 24-well culture plates were used with a neuron density of $2.5 \times 10^{5}$ cells per well. Neurons of $14-17 \mathrm{~d}$ in vitro (DIV) were used for experiments. For immunostaining, hippocampal neurons were seeded on glass coverslips in 12 -well plates with a density of $1.25 \times 10^{5}$ cells per well. To assess axonal development and synapse formation, hippocampal neurons at 0 DIV and 21 DIV, respectively, were treated with $2 \mu \mathrm{M}$ NIMoE (novel interaction motif E) for $3 \mathrm{~d}$ (media were changed daily to maintain peptide concentration relatively constant).
HEK 293 cells were maintained in DMEM (Invitrogen) supplemented with $10 \%$ fetal bovine serum and $1 \%$ penicillin/streptomycin. For protein expression in cell lines, cells were transfected with Calcium Phosphate Transfection reagent (Promega) and used $24 \mathrm{~h}$ later as described previously (Yang et al., 2009). The JNK inhibitor L-JNKI was purchased from Alexis Biochemicals. Thrombin was obtained from BIOPUR. Other assay reagents without indication were obtained from Sigma.

Plasmids and antibodies. The GST-tagged full-length and fragments of zD17 constructs were created by cloning zD17 and fragment cDNA sequences into a pGEX-4T-1 vector with a myc epitope sequence at the $\mathrm{N}$ terminus. The GSTJNK3 construct was cloned into the same vector. myc-tagged fragments of $\mathrm{zD} 17$ were also cloned into pcDNA3 to generate expression constructs. GFP-JNK1 and GFP-JNK2 expression constructs were created by cloning cDNA sequences into pEGFP-C1 plasmids. The vectors encoding myc-zD13, myc-zD15, myc-zD17, myc-zD17 zD23, GFP-PSD-95, GFP-PSD-95 ${ }^{\mathrm{C} 3,5 \mathrm{~S}}$, GFPSNAP25, and HA-SYTI were generously provided by Dr. El-Husseini's laboratory. The GFP-JNK3 construct was a gift from Dr. Vsevolod V. Gurevich (Vanderbilt University, Nashville, TN) (Song et al., 2006). The constructs expressing FLAG-JNK3, FLAG-MKK3, FLAG-MKK4, FLAG-MKK6, FLAGMKK7wt, and FLAG-kiMKK7 were purchased from Addgene.

The primary antibodies used to detect JNK1, activated-caspase3, and FLAG (DDDDK) epitopes were obtained from Abcam. AntizD17, anti-synaptophysin, and anti-GFP were purchased from Sigma. Anti-GST and antiTaul were purchased from Abm and Millipore, respectively. The antibody against postsynaptic density 95 (PSD-95) was obtained from Synaptic Systems. Purified IgG was supplied by Jackson ImmunoResearch. All other primary antibodies were purchased from Cell Signaling Technology. Secondary antibodies were HRP-conjugated anti-mouse (Cell Signaling Technology), anti-rabbit (PerkinElmer Life and Analytical Sciences), anti-GFP (Santa Cruz Biotechnology), and anti-myc (Invitrogen). Fluorescent Alexa Fluor 488- and Alexa Fluor 568-conjugated anti-mouse IgG1, IgG2a, and anti-rabbit IgG were obtained from Invitrogen.

Protein extraction and immunoblotting. The protocols for protein extraction have been described previously (Yang et al., 2009). For coimmunoprecipitation, cultured cells were washed with ice-cold PBS, followed by incubation with $0.5 \mathrm{ml}$ of gentle lysis buffer (GLB) containing $25 \mathrm{~mm}$ Tris-HCl, pH 7.4, 2 mм EDTA, 1 mm EGTA, 10 m $\mathrm{NaCl}$, 0.5\% Triton $\mathrm{X}-100$, and $10 \%$ glycerol supplemented with the Complete Protease Inhibitor Tablets (Roche Applied Science) and $1 \mathrm{~mm}$ PMSF. Total protein concentration was determined with the Bio-Rad Protein Assay kit. Lysates were first precleaned by incubating with protein A-Sepharose beads (Roche Applied Science) for $1 \mathrm{~h}$ at $4^{\circ} \mathrm{C}$, followed by incubation with indicated antibodies $\left(>16 \mathrm{~h}\right.$ at $\left.4^{\circ} \mathrm{C}\right)$ and protein A-Sepharose beads $(2 \mathrm{~h}$ at $4^{\circ} \mathrm{C}$ ). Lysates of rat brain tissues were precleaned with IgG and protein A beads. Immunoprecipitates were washed three times with GLB, boiled in $2 \times$ loading buffer with $1 \mathrm{~mm}$ dithiothreitol (DTT) for $3 \mathrm{~min}$, and analyzed with SDS-PAGE as described previously (Yang et al., 2009). The relative intensities of the bands on immunoblots were analyzed and quantified using NIH Image J. 
LDH assays and cell death detection. Neuronal cultures were challenged with indicated concentrations of NMDA for $1 \mathrm{~h}$, followed by $24 \mathrm{~h}$ survival. The release of $\mathrm{LDH}$ was measured with an in vitro toxicology assay kit (Sigma) according to the instructions of the manufacturer. Spectrophotometric measurement was performed on a Multilabel Plate Reader (Envision 2103; PerkinElmer Life and Analytical Sciences). The LDH reading represents the primary absorbance at a wavelength of $490 \mathrm{~nm}$ after subtraction of background absorbance at $690 \mathrm{~nm}$. The cell death level (percentage of total cell death) was quantified by dividing the LDH readings of sample groups by the readings from cultures incubated with $1 \%$ triton for 15 min representing maximal LDH. After the LDH assay, propidium iodide (PI) (Sigma) was added to the medium at a final concentration of $1 \mu \mathrm{g} / \mathrm{ml}$ and neurons were stained for $30 \mathrm{~min}$ at $30^{\circ} \mathrm{C}$. After fixation with $4 \%$ paraformaldehyde, neurons were washed with $1 \times$ PBS and stained with Hoechst 33342 (Sigma). The fluorescence of PI and Hoechst were examined with laser microscopy and analyzed with NIH ImageJ.

Kinase assays. JNK activity was measured with an in vitro kinase assay as described previously (Khatlani et al., 2007). Briefly, cell lysates were prepared and JNK was immunoprecipitated with specific antibodies. The immunoprecipitates were then resuspended in $1 \times$ kinase buffer (Cell Signaling Technology) supplemented with $200 \mu \mathrm{M}$ ATP and $250 \mu \mathrm{g} / \mathrm{ml}$ GST-c-Jun (1-89) and incubated at $30^{\circ} \mathrm{C}$ for $30 \mathrm{~min}$. Reactions were terminated by the addition of SDS-PAGE sample buffer. Phosphorylated c-Jun was resolved by SDS-PAGE and detected with anti-phospho-c-Jun.

Affinity binding assay and peptide array. An affinity binding assay was used to assess direct interaction between JNK3 and zD17. GST-fused full-length $\mathrm{zD} 17$ (GST-zD17 ${ }^{1-632}$ ), zD17 fragments (GST-zD17 ${ }^{1-310}$, GST-zD17 ${ }^{405-479}$, GST-D17 ${ }^{550-632}$, GST-zD17 ${ }^{125-140}$, GST-zD17 $7^{140-190}$, GST-zD17 ${ }^{190-210}$ ), and JNK3 (GST-JNK3) were purified from Escherichia coli BL21 with glutathione Sepharose 4B (GE Healthcare). Purified GST fusion proteins were resolved on SDS-PAGE and transferred onto nitrocellulose membranes. To prepare bait proteins, purified GST-myczD17 and GST-JNK3 were digested with thrombin overnight at room temperature, followed by clearance with $\beta$-aminobenzamidine-agarose (Sigma) for $1 \mathrm{~h}$ at $4^{\circ} \mathrm{C}$. The bait proteins were then prepared in affinity binding buffer [TBS with Triton X-100 (TBST) with 5\% skim milk and $4 \%$ sucrose] at a concentration of $10 \mu \mathrm{g} / \mathrm{ml}$. After blocking with affinity binding buffer at room temperature for $4 \mathrm{~h}$, the membrane was incubated with bait proteins overnight at $4^{\circ} \mathrm{C}$, and washed three times with TBST. Bound bait proteins were detected with primary antibody against myc epitope or JNK3 and HRP-conjugated secondary antibody. For mapping detailed interaction motifs, a peptide spot array was synthesized by PepMetric Technologies. The array contained overlapping peptides (15-mer peptides with 5 aa shift) to cover the N-terminal cytosolic domain of $\mathrm{zD} 17\left(\mathrm{zD} 17^{1-325}\right)$. The array membrane was initialized by washing twice with methanol for $10 \mathrm{~min}$ at room temperature, followed by three washes with TBST. The conditions for the preparation of array membrane, incubation with purified JNK3, and detection of bound bait protein were the same as those of the affinity binding assays.

Mitochondrial fractionation. Cultured neurons were lysed on ice for 10 min in TEEN mitochondria extraction buffer containing $20 \mathrm{~mm}$ Tris$\mathrm{HCl}$, pH 7.5, $10 \mathrm{~mm} \mathrm{KCl}, 1.5 \mathrm{~mm} \mathrm{MgCl}_{2}$, 1 mм EGTA, 1 mm EDTA, and 1 mм DTT supplemented with the Complete Protease Inhibitor tablets. The lysates were homogenized with a $28^{1 / 2}$ gauge syringe for a total of eight times on ice, followed by centrifugation twice at $700 \times g$ for $10 \mathrm{~min}$ at $4^{\circ} \mathrm{C}$. The supernatant was then centrifuged at $10,000 \times g$ for $30 \mathrm{~min}$ at $4^{\circ} \mathrm{C}$ to enrich mitochondria in the pellet. The cytosolic fraction was collected from the supernatant after additional centrifugation at $100,000 \times$ $g$ for $1 \mathrm{~h}$ at $4^{\circ} \mathrm{C}$. One and $4 \times$ lysis buffer were added to the mitochondrial and cytosolic fractions, respectively. Equivalent amounts of samples were resolved by SDS-PAGE and blotted with antibodies against cytochrome $c$ (Cyt $c$ ) and Bax.

Immunostaining and laser microscopy. Cultured hippocampal neurons on glass coverslips were used for immunostaining. After treatments indicated in text, neurons were fixed with $2 \%$ paraformaldehyde for $3 \mathrm{~min}$ at room temperature, followed by permeabilization with ice-cold methanol for $10 \mathrm{~min}$ at $-20^{\circ} \mathrm{C}$. The coverslips were then washed three times with PBS and immunostained with appropriate antibodies dissolved in
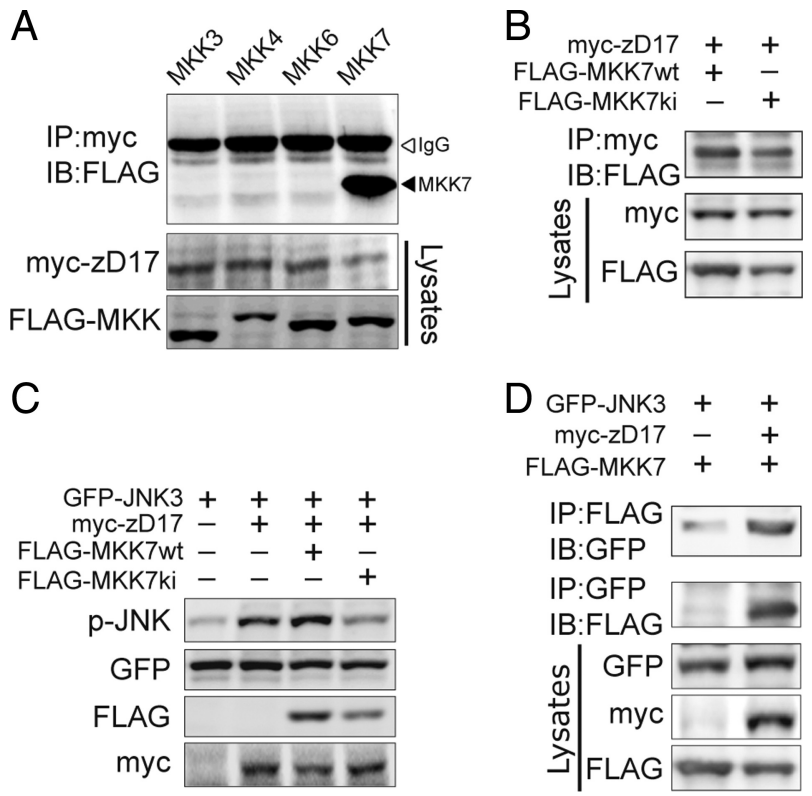

Figure 2. Formation of the JNK-ZD17-MKK7 signaling module. $A$, ZD17 selectively interacts with MKK7. Only FLAG-MKK7 is present in immunoprecipitates. $\boldsymbol{B}, \mathrm{ZD} 17$ interacts with MKK7 wild-type (wt) and the kinase-inactive mutant (ki). C, MKK7 contributes to the zD17-mediated phosphorylation of JNK3. Coexpression of FLAG-MKK7ki in HEK293 cells reduces the phosphorylation level of GFP-JNK3 promoted by myc-zD17 (fold change to JNK3 only; JNK3 + zD17, $2.28 \pm 0.23 ;$ JNK3 + zD17 + MKK7wt, $3.10 \pm 0.43 ;$ JNK3 + zD17 + MKK7ki, $1.48 \pm 0.22$, $p<0.01$ compared with JNK3 + zD17). D, zD17 recruits MKK7 and JNK3 in a signaling module. GFP-JNK3 and FLAG-MKK7 are expressed in the HEK293 cells, with or without myc-zD17. The presence of MKK7 in the JNK3 immunoprecipitates is enhanced by coexpression of ZD17 (fold change to JNK3 + MKK7; JNK3 + zD17 + MKK7, $2.41 \pm 0.17, p<0.01$ ). IP, Immunoprecipitation; IB, immunoblot.

PBS for $1 \mathrm{~h}$ at room temperature. After washing three times with PBS, coverslips were incubated with Alexa Fluor-labeled secondary antibodies from Invitrogen for an additional $1 \mathrm{~h}$. To assess axonal development, hippocampal cultures were stained with anti-Tau- 1 antibody and DAPI. For mitochondrial detection, neuronal cultures were incubated at $37^{\circ} \mathrm{C}$ for $30 \mathrm{~min}$ with $2 \mu \mathrm{M}$ MitoTracker-TR (Invitrogen). Coverslips were then fixed and mounted with ProLong Gold Antifade Reagent (Invitrogen). Fluorescence was captured using a Carl Zeiss Observer Z1 microscope, and all images were analyzed with NIH ImageJ and processed using Adobe Photoshop (Adobe Systems).

Transient ischemia in rats. Adult male Sprague Dawley rats weighing 250-290 g were used for transient middle cerebral artery occlusion (MCAo). Rats were anesthetized with 5\% isoflurane and maintained with $2 \%$ isoflurane in $70 \% \mathrm{~N}_{2} \mathrm{O}$ and $30 \% \mathrm{O}_{2}$ using a face mask. Ketoprofen at $5 \mathrm{mg} / \mathrm{kg}$ was given before surgery to block pain. Rectal temperature was maintained at $37^{\circ} \mathrm{C}$ during surgery with a homeothermic blanket system (Harvard Apparatus). The scalp was incised at the midline and the skull was exposed. The skull was thinned with a dental drill at a region ipsilateral to the expected ischemia zone $(2 \mathrm{~mm}$ posterior and $5 \mathrm{~mm}$ lateral from the bregma). A laser probe of the Doppler flowmeter (Perimed) was placed at this location during surgery to detect cerebral blood flow (CBF) (Nagel et al., 2011). We induced transient MCAo using a method of intraluminal vascular occlusion described previously (Longa et al., 1989; Belayev et al., 1996; Aarts et al., 2002). Briefly, a poly-L-lysinecoated 3-0 monofilament nylon suture (Harvard Apparatus) with a rounded tip was advanced from the left common carotid artery into the internal carotid artery until a sudden drop of CBF was noted, indicating the blockade of the origin of the MCA. The success of induction of ischemia was confirmed by a fall of $\mathrm{CBF}$ to $<25 \%$ of baseline level and by neurological assessment scores during MCAo ( $1 \mathrm{~h}$ after the onset). Two hours after MCAo, rats were reanesthetized, and the suture was removed to allow reperfusion, confirmed by the increase of $\mathrm{CBF}$ at the same area. 
A

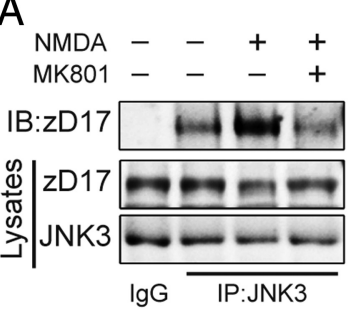

B
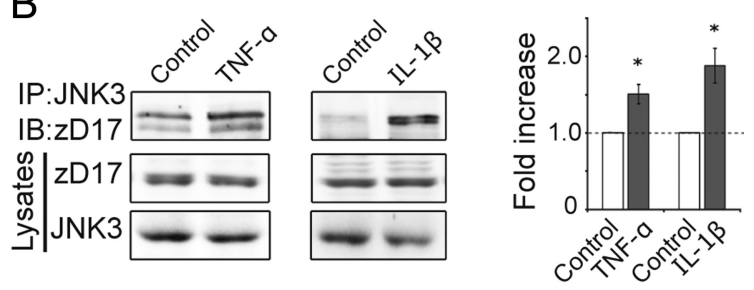

C

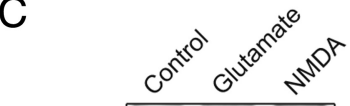

IP:JNK1

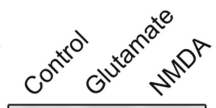

IP:JNK2 , ,

IP:JNK3
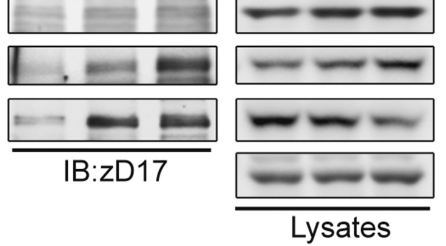

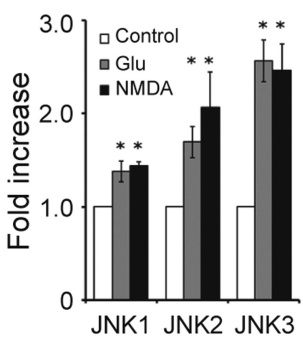

E

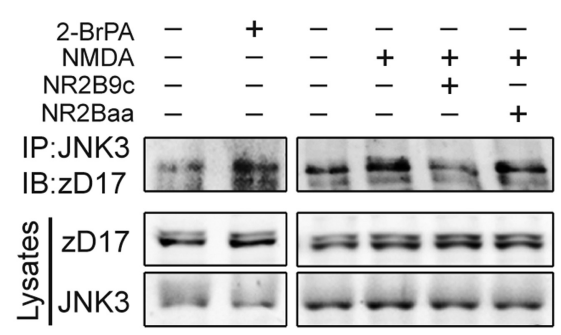

Figure 3. Regulation of the ZD17-JNK3 signaling module in response to NMDA-induced excitotoxicity. $\boldsymbol{A}$, NMDA-induced excitotoxicity promotes zD17-JNK3 interaction in neuronal cultures. NMDA stimulation enhances the ZD17-JNK interaction assessed by coimmunoprecipitation (fold change compared with control; NMDA, $1.49 \pm 0.09, p<0.01$; NMDA + MK801, $0.96 \pm$ $0.14, p=0.78) . B$, The cytokines TNF- $\alpha$ and IL- $1 \beta$ enhance the zD17-JNK3 interaction in neuronal cultures. TNF- $\alpha(50 \mathrm{ng} / \mathrm{ml}, 6 \mathrm{~h})$ and IL-1 $\beta(10 \mathrm{ng} / \mathrm{ml}, 16 \mathrm{~h})$ treatments induced a $1.53 \pm 0.11$ - and $1.88 \pm 0.23$-fold increase of zD17-JNK3 interaction, respectively. $C$, Glutamate and NMDA enhance the zD17 interaction with JNK isoforms 2 and 3 . The enhancement on JNK2 (fold increase compared with control; glutamate, $1.7 \pm 0.2 ; \mathrm{NMDA}, 2.1 \pm 0.4$ ) and on JNK3 (glutamate, $2.6 \pm 0.2 ; \mathrm{NMDA}, 2.5 \pm 0.3$ ) are higher than that on JNK1 (glutamate, $1.4 \pm 0.1 ;$ NMDA, $1.4 \pm 0.1$ ). D, Binding to PSD-95 interferes with the zD17-JNK3 interaction (fold change; PSD-95, $0.82 \pm 0.03, p<0.01$; PSD-95 ${ }^{\mathrm{C}, 55}, 1.25 \pm 0.07, p<0.05$ ) and reduces JNK3 phosphorylation (fold change; PSD-95, $0.64 \pm 0.11, p<0.05$; PSD-95 ${ }^{\mathrm{C3}, 55}, 1.19 \pm 0.21, p=0.42$ ) in HEK293 cells. $\boldsymbol{E}$, In neuronal cultures, blocking palmitoylation with 2-BrPA (100 $\mu \mathrm{M}, 6 \mathrm{~h}$ ) promotes the ZD17-JNK3 interaction (fold change; 2-BP, $1.59 \pm 0.05$ ). Disrupting the NR2B-PSD-95 association with NR2B9C (1 $\mu \mathrm{M}, 30 \mathrm{~min}$ before NMDA treatment) reduces the NMDA excitotoxicityinduced zD17 interaction with JNK3, examined $15 \mathrm{~min}$ after NMDA challenge (fold change; NMDA, $1.64 \pm 0.04$; NR2B9c, $1.12 \pm$ 0.1 ; NR2Baa, $1.48 \pm 0.1, p=0.23$ to NMDA). $t$ test. ${ }^{*} p<0.05$ and $^{* *} p<0.01$. Error bars show means \pm SEM. IP, Immunoprecipitation; IB, immunoblot.

Peptide administration. Peptides were synthesized by PepMetric Technologies or GL Biochem. Peptides were prepared freshly in saline on the day of experiment at a stock concentration of $1 \mathrm{mg} / \mathrm{ml}$. Peptides at 1 $\mathrm{mg} / \mathrm{kg}$ were administrated at the indicated times by a single intravenous injection into the rat tail. Scrambled NIMoE (NIMoEscr) or short NIMoE (NIMoEsh) were injected at $4 \mathrm{~h}$ after MCAo onset to randomly chosen rats. For long-term studies, peptides were given $2 \mathrm{~h}$ after MCAo onset. We injected NIMoEscr and NIMoEsh, assessed brain damage, and performed functional evaluations in a double-blind manner. To examine the delivery of the peptide into the brain, either $5 \mathrm{mg} / \mathrm{kg}$ FITC-labeled NIMoE or saline was intravenously injected $1 \mathrm{~h}$ before perfusion. The rats were then perfused with PBS followed by fixation solution (4\% paraformaldehyde in PBS). Brains were removed, postfixed in fixation solution for $2 \mathrm{~h}$, and then soaked in $20 \%$ sucrose-PBS buffer at $4^{\circ} \mathrm{C}$ for $24 \mathrm{~h}$. After rapid freezing in Tissue-Tek embedding medium (Sakura) on dry ice, $20 \mu \mathrm{m}$ sections were cut on a cryostat (Leica CM3050) and examined for FITC fluorescence by laser microscopy (Carl Zeiss Axiovert 200).

Histological assessment. Rats were allowed to survive for $24 \mathrm{~h}$. After decapitation, the brains were immediately removed and serially sec- tioned in the coronal plane at a thickness of 2 $\mathrm{mm}$ with a slicer matrix (Zivic). A total of eight sections were collected and then incubated in PBS with 1\% 2,3,7-triphenyltetrazolium chloride at $37^{\circ} \mathrm{C}$ for $10 \mathrm{~min}$. The stained slices were then fixed in $4 \%$ paraformaldehyde and digitized with a color flatbed scanner (MFC8860DN; Brothers). The infarct area within each section was traced and quantified using $\mathrm{NIH}$ ImageJ. The infarct volume was calculated using the following formula: $V=d(A 1+$ $A 2+\ldots+A 8)$, where $V$ is the infarct volume (cubic millimeters), $d$ is the distance between sections, and $A$ is the infarct area within each section.

Functional tests. All animals were tested for neurological function during ( $1 \mathrm{~h}$ after onset) and $24 \mathrm{~h}$ after MCAo. We evaluated motor, sensory, and coordination capacities using modified neurological severity scores (Bederson et al., 1986; Watanabe et al., 2004). The motor system tests comprised seven components, including motor initiation test, free activity, posture, walking, tail suspension, hindlimb flexion, and pushing test. Somatosensory tests included a tactile test and forelimb placing test. Coordination functions were assessed with three tests, including a foot fault test, edge test, and balance beam. Performance in the tests was evaluated on a cumulative scale from 0 to 2 (10 tests), except for two tests (tail suspension and balance beam) in which scores ranged from 0 to 3 and 0 to 4 , respectively. The scores from each test were summed and represented as a single overall neurological score ( 0 to 27). The higher the score, the greater is the functional impairment. The adhesive-removal test (ART) was used for additional evaluation of somatosensory deficits (Bouet et al., 2009). An adhesive tape strip was securely wrapped around the wrist of the right forepaw. The rat was returned to the cage, and its behavioral attempts to remove the tape strip by either shaking its paws and/or bringing its paws to its mouth was monitored and counted within total $2 \mathrm{~min}$. Because an intact and correct sensory system is required for sensing the strip, the shorter ART time represents the sensory impairment, whereas longer ART time (usually 2 min) represents a completely intact function.

Statistics. All values in text and figures are presented as mean \pm SEM. Student's $t$ test or one-way ANOVA was performed using Excel software (Microsoft). The limit of statistical significance was set at a $p$ value $<0.05$.

\section{Results}

JNK activation is regulated by the $\mathrm{zD} 17-\mathrm{MKK} 7-\mathrm{JNK}$ signaling module

To examine the impact of PATs on JNK activation, we expressed several neuronal PATs individually with JNK3 in HEK293 cells. Under both resting and osmotic stress conditions, zD17 greatly facilitated JNK3 phosphorylation, whereas other tested PATs showed no effects (Fig. $1 A$ ). The kinase activity of JNK3 was also enhanced by expression of zD17 (Fig. $1 B$ ). We further examined the involvement of the PAT activity of zD17 by using a PAT activity-deficient mutant of zD17 (zD17 $\Delta$ ) (Huang et al., 2004). Similar to wild-type zD17, zD17 $\Delta$ strongly enhanced JNK3 phosphorylation (Fig. $1 C$ ). These results indicate that $\mathrm{zD} 17$ is selec- 
tively involved in activating JNK in a PAT activity-independent manner. Because zD17 does not have a canonical kinase domain, we asked whether zD17 might interact directly with JNK and modulate its activity. Using coimmunoprecipitation, we found that JNK3 associated with zD17 but not with other similar PATs (zD15 and zD20) (Fig. 1D). This association of JNK3 was independent of the PAT activity of zD17, because both zD17 wild-type and zD17A immunoprecipitated JNK3 (Fig. $1 E)$. Similar to JNK3, JNK1 and JNK2 are able to interact with $\mathrm{zD} 17$ when expressed in HEK293 cells (Fig. $1 F$ ). In vitro, purified JNK3 was capable of binding purified myc-zD17 (Fig. 1G). Thus, zD17 is a binding partner of JNK.

Because zD17 promoted JNK phosphorylation and activity (Fig. $1 B$ ), we examined the role of several mitogenactivated protein kinase kinases (MAP$\mathrm{KKs}$ ), upstream activators of JNK, in a potential signaling module (Weston and Davis, 2007). When MAPKKs were expressed in HEK293 cells, zD17 was able to coimmunoprecipitate the JNK activator MKK7. In contrast, the JNK activator MKK4 and the p38 activators MKK3 and MKK6 did not associate with zD17 (Fig. $2 A$ ). To further confirm the functional involvement of MKK7, we used kinaseinactive MKK7 (MKK7ki) (Merritt et al., 1999). Because MKK7ki interacted with zD17 similar to MKK7 wild type (Fig. $2 B$ ), we expressed MKK7ki as a dominant negative, resulting in attenuated JNK3 phosphorylation (Fig. 2C). Moreover, the association of JNK3 and MKK7, assessed by coimmunoprecipitation, was strongly enhanced in the presence of zD17 (2.41 \pm 0.17 -fold, $p<0.01$ ) (Fig. $2 D$ ). These results indicate that $\mathrm{zD} 17$ recruits MKK7 and JNK to form a signaling module for JNK activation.
A
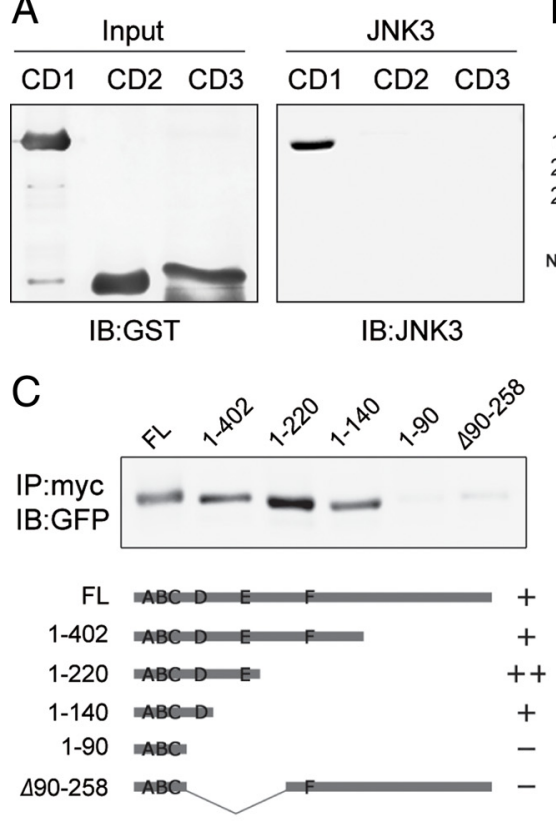

B

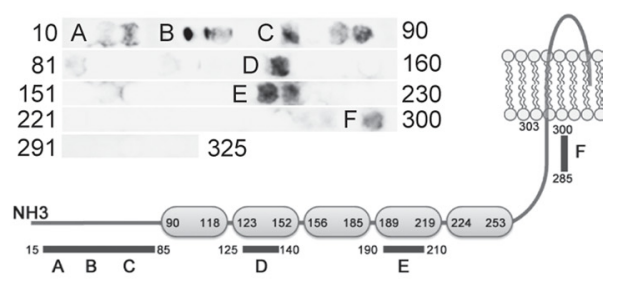

D

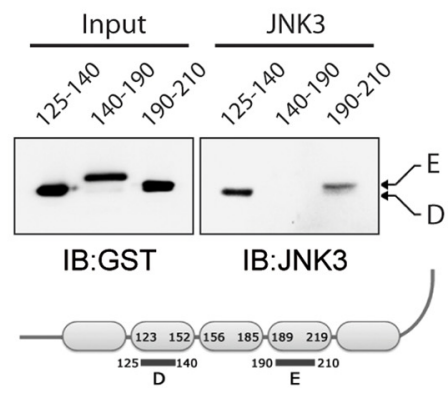

E

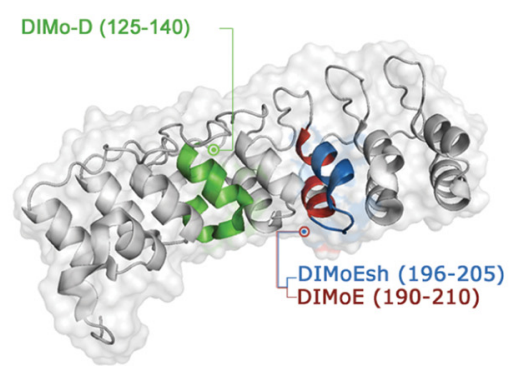

Figure 4. Identification of novel JNK-interacting motifs on ZD17. A, JNK-interacting motifs are embedded at the $\mathrm{N}$ terminus of ZD17. Purified GST-tagged zD17 fragments are its cytosolic domains (CD1, zD17 ${ }^{1-310} ;\left(\mathrm{CD} 2, \mathrm{zD} 17^{405-479} ; \mathrm{CD} 3, \mathrm{ZD} 17^{550-632}\right) . \boldsymbol{B}$, The in vitro peptide scan of potential JNK-interacting motifs on zD17 N-terminal cytosolic domain. Interaction-positive regions are plotted on the diagram showing the N-terminal part of zD17. Bold black lines with marks $(\boldsymbol{A}-\boldsymbol{F})$ indicate the potential JNKinteracting motifs from the peptide scan, and their locations on ZD17 are labeled beside the lines. The ankyrin domains for protein interaction were plotted as rounded rectangles with locations indicated. C, Two motifs located in different ankyrin domains are critical for the ZD17-JNK interaction. myc-tagged full-length ZD17 (FL) and fragments of ZD17 with assorted deletions are indicated at bottom, and embedded potential interaction motifs $(\boldsymbol{A}-\boldsymbol{F})$ are labeled. The interaction ability (plus or minus) of these constructs is plotted. $\boldsymbol{D}$, JNK3 binds to motif D and motifE on ZD17. MotifD, motifE, and the third ankyrin repeat between (as shown in diagram) were fused with GST and purified. After incubation with JNK3, only motif D and motif Ebound to JNK3. E, The locations on zD17 from which peptides NIMoD, NIMoE, and NIMoEsh are derived, shown in a simulated structure of ZD17 ankyrin repeats. 3D structure data was obtained from National Center for Biotechnology Information (Protein Data Bank accession number 3EU9) and processed with PyMOL software (version 1.3). IP, Immunoprecipitation; IB, immunoblot.

\section{Neuronal excitotoxicity promotes} formation of the $\mathrm{zD} 17-\mathrm{JNK}$ module

As an important transducer of stress signals in the neuron, JNK is activated in response to various stresses such as excitotoxicity and inflammation (Borsello and Forloni, 2007). We thus asked whether the $\mathrm{zD} 17-\mathrm{JNK}$ signaling module is recruited in these scenarios. Inflammation induced by the cytokines tumor necrosis factor- $\alpha$ (TNF- $\alpha$ ) or interleukin-1 $\beta$ (IL-1 $\beta$ ), as well as excitotoxicity induced by glutamate or NMDA stimulation robustly promoted the zD17-JNK3 interaction in cortical neuronal cultures (Fig. 3A,B). Moreover, compared with stress-responsive isoforms JNK2 and JNK3, the JNK1-zD17 interaction was less sensitive to either NMDA or glutamate treatment (Fig. 3C). Because excitotoxicityinduced JNK activation is predominantly mediated by MKK7 (Centeno et al., 2007), we focused on excitotoxicity as our cellular model to study mechanisms underlying activation of the zD17-JNK signaling module and its contribution to neuronal cell death.
We examined the activation pathways by which the zD17JNK signaling module is regulated by excitotoxicity. zD17 has been shown to interact with many different substrates, and it may be that changes in interaction of $\mathrm{zD} 17$ with one or more of its substrates affects its availability for interaction with JNK3 (Huang et al., 2009). One substrate of zD17, PSD-95 is especially relevant, because excitotoxicity has been shown to alter its palmitoylation and protein-interaction profile (Kornau et al., 1995; El-Husseini et al., 2002; Kang et al., 2008). In HEK293 cells, overexpression of PSD-95 impeded the zD17-JNK3 interaction and JNK3 phosphorylation (Fig. 3D). This impact of PSD-95 was completely abolished by mutating its palmitoylation sites, which eliminated the interaction with zD17 (Huang et al., 2009). We also used a broad-spectrum palmitoylation inhibitor, 2-bromopalmitate (2-BrPA), to inhibit the $\mathrm{zD} 17$ interaction with its substrates in cultured neurons (Huang et al., 2009; Yang 
A

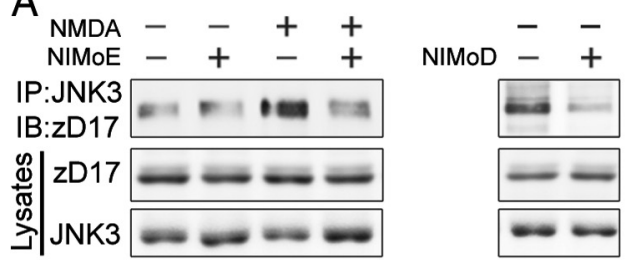

B

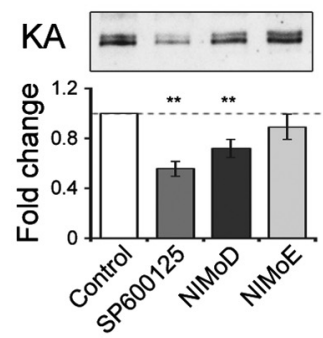

C

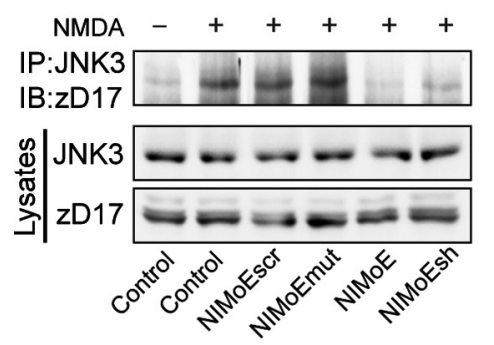

$\mathrm{E}$

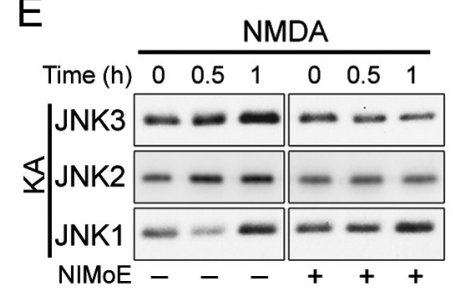

D
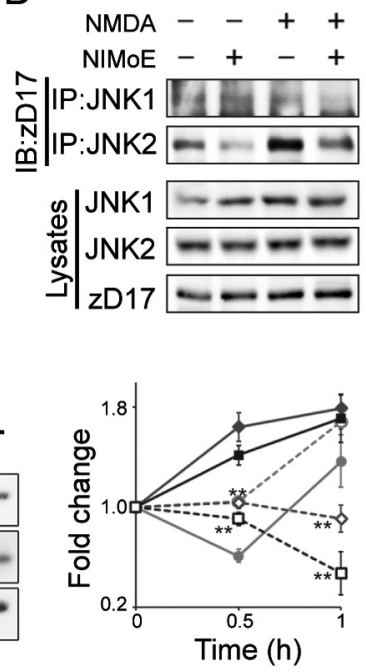

Figure 5. Isoform- and scenario-selective inhibition of JNK by peptides derived from JNK binding motifs on ZD17. A, NIMoE blocks the NMDA-induced enhancement of the ZD17-JNK interaction without affecting baseline interaction in neuronal cultures (normalized to control; NMDA, $173.6 \pm 16.9 \%, p<0.05 ; \mathrm{NMDA}+\mathrm{NIMoE}, 106.5 \pm 1.2 \%, p=0.16 ; \mathrm{NIMOE}, 103.5 \pm 6.2 \%, p=$ $0.64 ; \mathrm{NIMOD}, 54.1 \pm 16 \%, p<0.01) . \boldsymbol{B}$, The effect of peptides on JNK3 basal activity. JNK3 is immunoprecipitated from cell lysates, followed by the detection of kinase activity with a kinase assay (KA). Pretreating cells with a known JNK inhibitor, SP600125 (10 $\mu \mathrm{M})$, or NIMoD $(1 \mu \mathrm{M})$ for $2 \mathrm{~h}$ caused a significant (fold change compared with control, $0.56 \pm 0.06)$ or partial $(0.72 \pm 0.07)$ reduction of JNK basal activity, respectively. NIMoE $(1 \mu \mathrm{M})$ has no significant impact on basal activity of JNK3 $(0.89 \pm 0.10, p=$ 0.33). C, NIMoE and NIMoEsh, but not control peptides NIMoEscr and NIMoEmut, blocks the NMDA-induced enhancement of the zD17-JNK3 interaction (normalized to control; NMDA, $240.9 \pm 3.4 \%$; NMDA + NIMoEscr, $226.4 \pm 27.1 \%, p=0.62$ compared with NMDA; NMDA + NIMoEmut, $208.7 \pm 50.5 \%, p=0.56 ; \mathrm{NMDA}+\mathrm{NIMoE}, 109.3 \pm 11.2 \%, p<0.01 ; \mathrm{NMDA}+$ NIMoEsh, $105.7 \pm 9.5 \%, p<0.01)$. D , The effect of the peptide NIMoE on the zD17-JNK1/2 interaction. $E$, NIMoE abolishes NMDA-induced activation of JNK2 and JNK3 but not JNK1. JNK is immunoprecipitated from the cell lysate, followed by the determination of JNK activity by kinase assays (KA) (normalized to $0 \mathrm{~h}$ JNK1) (filled circle with solid line): $0.5 \mathrm{~h}, 61.0 \pm 5.4 \%$ and $1 \mathrm{~h}, 137.8 \pm 20.8 \%$; JNK1 + NIMoE (open circle with dotted line): $0.5 \mathrm{~h}, 104.3 \pm 2.6$ and $1 \mathrm{~h}, 168.5 \pm 3.3 \%$; JNK2 (filled diamond with solid line): $0.5 \mathrm{~h}, 164.3 \pm 11.5 \%$ and $1 \mathrm{~h}, 179.2 \pm 11.2 \%$; JNK2 + NIMoE (open diamond with dotted line): $0.5 \mathrm{~h}, 103.7 \pm 5.0 \%$ and $1 \mathrm{~h}$, $90.9 \pm 10.9 \%$; JNK3 (filled square with solid line), $0.5 \mathrm{~h}, 141.7 \pm 7.8 \%$ and $1 \mathrm{~h}, 172.1 \pm 18.9 \%$; JNK3 + NIMoE (open square with dotted line), $0.5 \mathrm{~h}, 90.6 \pm 5.3 \%$ and $1 \mathrm{~h}, 47.0 \pm 17.6 \%$ ). $t$ test. ${ }^{* *} p<0.01$. Line graphs show means \pm SEM. IP, Immunoprecipitation; IB, immunoblot.

et al., 2009). This treatment robustly enhanced the zD17-JNK3 interaction (Fig. 3E). Excitotoxicity has been shown to mobilize PSD-95 to NMDA receptors (NMDARs), which initiates neurotoxic signaling (Kornau et al., 1995; Aarts et al., 2002). A previously described peptide, NR2B9c, was applied to block the PSD95-NMDAR association (Aarts et al., 2002). This peptide, but not its mutated control NR2Baa, also impeded the enhancement of the $\mathrm{zD} 17-\mathrm{JNK} 3$ interaction (Fig. 3E). Together, these results suggest that the PSD-95 contributes, at least partially, to the regulation of the $\mathrm{zD} 17-\mathrm{JNK}$ interaction in response to excitotoxicity.

\section{Identification of JNK binding motifs on $\mathrm{zD} 17$}

To develop blockers of the zD17-JNK interaction, we first identified two novel JNK binding motifs on zD17. We purified three cytosolic domains (CD1, CD2, and CD3) of zD17 with a GST tag

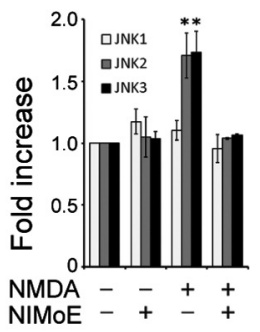

$\rightarrow-J N K 1$

-o- JNK1+NIMOE $\rightarrow$ JNK2 - - JNK2+NIMOE $\rightarrow$ JNK3 - - JNK3+NIMoE and found that, in vitro, JNK3 binding predominantly occurred at CD1 (Fig. $4 A)$. To obtain additional information, we used a peptide array containing 15mers with a 5 aa shift to cover CD1 and identified regions on the membrane to which JNK3 bound (Fig. 4B). Six potential binding regions in three categories were detected: $\mathrm{N}$ terminus (motifs $\mathrm{A}-\mathrm{C}$ ), ankyrin repeats (motifs D and E), and submembrane (motif F). These zones are merely candidate regions and may not reflect actual binding domains. Thus, we generated a series of zD17 deletion mutants and examined their capability of interacting with JNK3 by coimmunoprecipitation (Fig. 4C). Deleting CD2 and CD3 did not affect the zD17-JNK3 interaction. Deletion of motif F did not reduce, but instead increased, the coimmunoprecipitation of JNK3. Removing motif E diminished the interaction. Strikingly, further deleting motif $\mathrm{D}$, or selectively removing motifs D and E, from full-length zD17 completely eliminated the interaction (Fig. 4C), suggesting the involvement of these two motifs in binding JNK3. Motifs $\mathrm{D}$ and $\mathrm{E}$ are located within the second and the fourth ankyrin repeats, respectively. Although zD17 contains five ankyrin repeats, our data indicate that JNK3 binds specifically to motifs D and E of $\mathrm{zD} 17$ with a preference for motif $\mathrm{D}$ (Fig. 4D).

\section{Isoform- and scenario-selective inhibition of JNK}

We next determined whether targeting these two motifs could inhibit the excitotoxicity-induced zD17-JNK interaction and JNK activation. We synthesized peptides comprising motif D (TPLHWATRGGHLSMV; NIMoD) and motif E (MTPLMWAAYRTHSVDPTRLL; NIMoE) and fused them to the cellmembrane transduction domain of the HIV-1 Tat protein to allow the peptides to penetrate the cell membrane (Schwarze et al., 1999). Based on results from the peptide array, we optimized NIMoE into a shorter 10-mer peptide (WAAYRTHSVD, NIMoEsh) that included primarily the loop region of motif $\mathrm{E}\left(\mathrm{zD} 17^{196-205}\right)$ (Fig. $4 E)$. BLAST searches showed that the sequence of NIMoE/NIMoEsh is found only within zD17 (data not shown), suggesting the specificity of these peptides. Bath application of NIMoD to neuronal cultures significantly diminished the coimmunoprecipitation of zD17 with JNK3, resulting in a reduction of JNK3 basal activity (Fig. 5A). NIMoE showed no effect on the baseline of the zD17-JNK3 interaction and JNK3 activity, but NIMoE, but not the scramble and mutated controls, selectively blocked the enhancement of the interaction and of JNK3 activity induced by NMDA (Fig. $5 B, C, E$ ). It also eliminated the enhancement of the JNK2-zD17 interaction and JNK2 activity (Fig. 5D,E). In con- 
trast, NIMoE did not inhibit the normal activation of JNK1 (Fig. 5E).

Because one concern with the use of pan-JNK inhibitors as therapeutics is their nonselective inhibition of JNK1, which is essential for normal neuronal development (Oliva et al., 2006), we tested the effect of NIMoE on axonal development, a process that is inhibited by SP600125 (anthra[1,9-cd]pyrazol-6(2H)-one). In contrast to results with this pan-JNK inhibitor (Fig. 6A), axon initiation and extension were not affected by chronic incubation with NIMoE in cultured neurons (Fig. 6B).

\section{Neurons are protected from excitotoxicity by targeting the zD17-JNK module}

JNK activation is critical for excitotoxicity-induced neuronal death (Centeno et al., 2007). Application of excitotoxic NMDA causes degeneration of neurites, nuclear condensation, membrane permeabilization, and $\mathrm{LDH}$ release in neuronal cultures (Fig. $7 A, B$ ). Pretreatment with NIMoE or NIMoEsh effectively preserved neuron morphology and significantly prevented excitotoxicityinduced cell death as measured by LDH release and PI staining, whereas NIMoEscr, mutant NIMoE (NIMoEmut), and a control peptide derived from an unrelated region of zD17 $\left({ }^{255}\right.$ NVKGESALDLAKQ ${ }^{267}$; DIPep1) showed no protective effects in neuronal cultures (Fig. $7 A-E$ ). NIMoE also protected neurons against a broad range of NMDA concentrations $(10-100 \mu \mathrm{M})$ (Fig. $7 F)$.

JNK2/3 activation mediates neuronal death via two major output pathways: by phosphorylating c-Jun to facilitate transcription of pro-death genes, and by activating the mitochondrial caspase-3 pathway to induce apoptosis (Weston and Davis, 2007). NIMoE and NIMoEsh were able to prevent NMDAinduced c-Jun phosphorylation (Fig. $8 A, B$ ). We found, furthermore, that caspase- 3 cleavage was also effectively blocked by NIMoE and NIMoEsh, whereas control peptides had no effect (Fig. 8C,D). JNK-mediated caspase-3 cleavage depends on translocation of the proapoptotic protein Bax to mitochondria and subsequent release of Cyt $c$ (Tsuruta et al., 2004). We found that NMDA-induced translocation of both Bax and Cyt $c$ was inhibited by NIMoE and NIMoEsh (Fig. $8 E, F$ ).

\section{Targeting the zD17-JNK module protects brains from ischemic stroke}

We next explored the potential of applying this strategy in vivo in a model of transient ischemic stroke (Longa et al., 1989). Adult male Sprague Dawley rats were subjected to left MCAo for $2 \mathrm{~h}$, followed by $22 \mathrm{~h}$ reperfusion (Fig. 9A). The zD17-JNK3 interaction in the injured hemisphere $(\mathrm{L})$ remained enhanced up to at least $6 \mathrm{~h}$ after the ischemic insult, implying a broad time window for potential intervention. Because of the consideration of its potential for therapeutic applications, we used the shorter form peptide NIMoEsh in our in vivo experiments. A single intravenous injection of NIMoEsh 30 min before MCAo effectively attenuated the enhancement of the zD17-JNK3 interaction and dramatically reduced the total infarct size (by $\sim 80 \%$ ) (Fig. $9 B-$ $D)$. We further assessed the effects of postsurgical interventions at
2 or 4 h. Compared with the infarct volume of nontreated, salinetreated, and scramble peptide-treated groups, NIMoEsh still showed effective protection for the ischemic brain (Fig. 9D). The behavioral deficits, as evaluated with neurological scores (Bederson et al., 1986; Watanabe et al., 2004), were substantially reduced by NIMoEsh administration (Fig. 9E). The quantitative ART further confirmed a large preservation of somatosensory functions in NIMoEsh-treated rats (Fig. 9F) (Bouet et al., 2009). The improvement of behavioral performance persisted over $14 \mathrm{~d}$ in ischemic rats injected with NIMoEsh $2 \mathrm{~h}$ after surgery, assessed in a double-blind manner (Fig. 9G,H). Together, these results suggest that targeting the $\mathrm{zD} 17-\mathrm{JNK}$ module to prevent JNK activation is effective in protecting the brain from ischemic injury and in improving behavioral outcomes.

\section{Discussion}

$\mathrm{zD} 17$ is a PAT that catalyzes a posttranslational lipid modification known as palmitoylation. Although much is known about the broad effects of palmitoylation on protein trafficking, stability, and function, our understanding of the pathophysiological roles of PATs in the nervous system remains poor (el-Husseini and Bredt, 2002; Bijlmakers and Marsh, 2003). In addition to its well-studied function in protein palmitoylation, we report here that PAT zD17 possesses a novel function that appears unique in the PAT family. Via its ankyrin domain, a domain used for protein-protein interaction and signaling, zD17 interacts with JNK and regulates neuronal cell death in acute ischemic stroke. By manipulating this novel zD17-JNK interaction, we provide a therapeutic strategy for acute ischemic brain injury with high efficacy and the potential for reduced side effects (Fig. 10).

Our in vivo and in vitro assays indicate that $\mathrm{zD} 17$ is a binding partner of JNK. One of the activators of JNK, MKK7, a stress mediator, is also selectively and functionally associated with zD17 (Figs. 1, 2). This suggests that MKK7-zD17-JNK forms a signaling module for JNK activation in response to stressors, including the ischemic stress that we report here. How are the multiple functions of $\mathrm{zD} 17$ connected to contribute to neuronal cell death? 


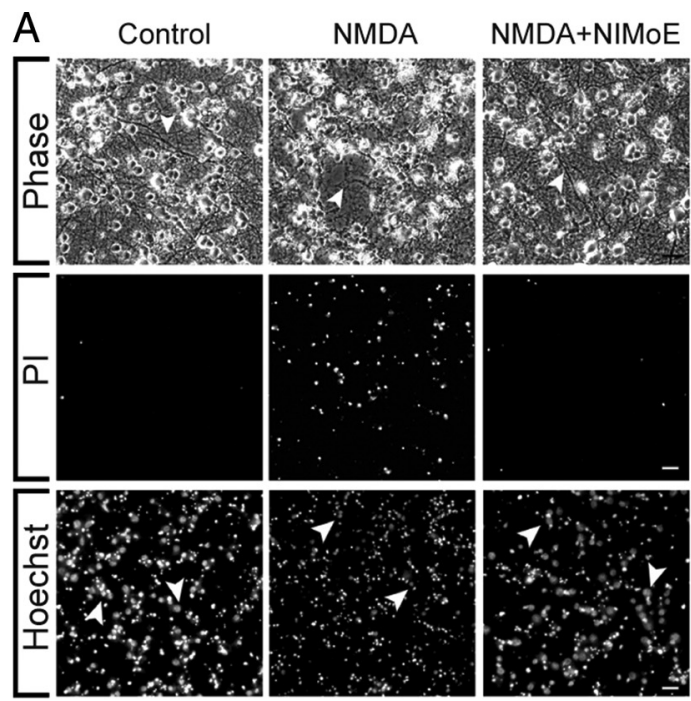

B

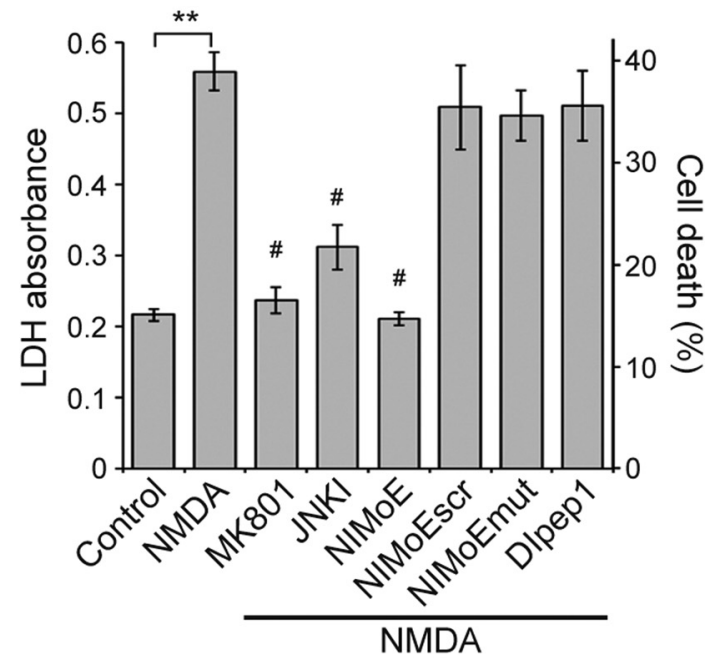

C

NMDA

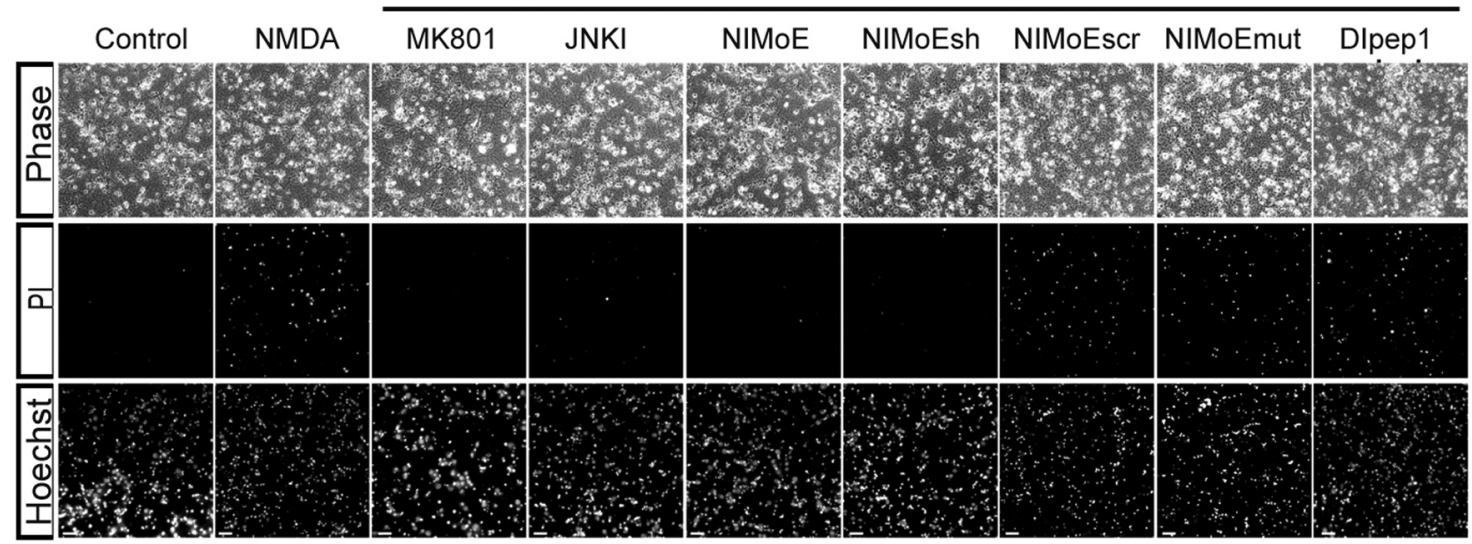

D

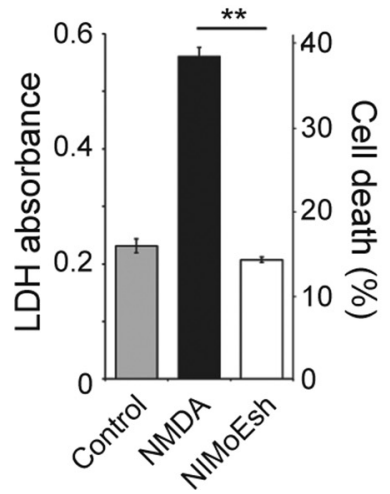

E

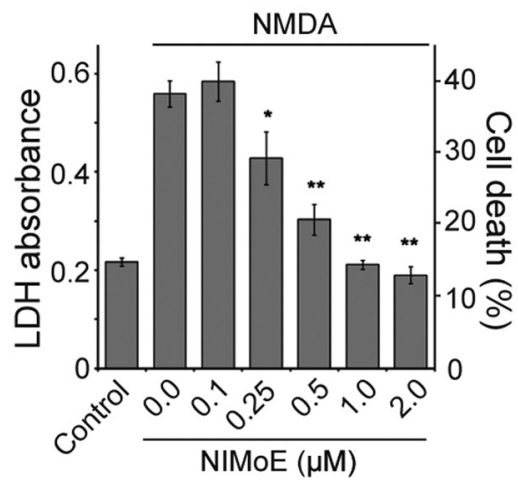

F

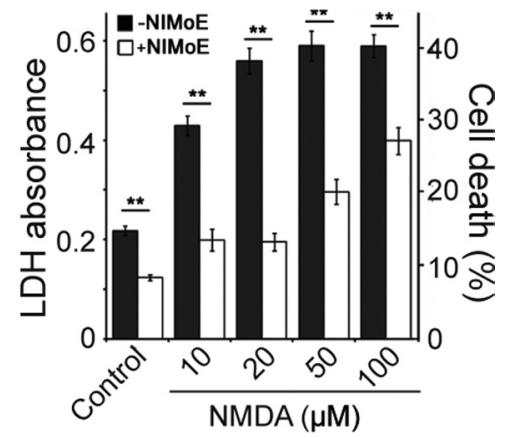

Figure 7. Neuroprotection against NMDA excitotoxicity by targeting the zD17-JNK interaction. $\boldsymbol{A}$, NMDA excitotoxicity-induced neuronal cell death is prevented by NIMoE. The phase contrast image, PI staining, and nuclear Hoechst staining are shown as indicated. The arrowheads indicate the integrity of neurites in the top panel (phase) and the shape of nucleus in the bottom panel (Hoechst). Scale bar, $20 \mu \mathrm{m}$. B, Detection of cell death with LDH assays. The cell death level was quantified by normalizing to total cell death (cultures treated with $1 \%$ Triton X-100). ${ }^{* *} p<0.01$; ${ }^{\#} p<0.01$ compared with NMDA alone. C, Effects of MK801 and peptides on NMDA excitotoxicity-induced neuronal cell death. Scale bar, $20 \mu \mathrm{m}$. $\boldsymbol{D}, \mathrm{Quantification}$ of LDH release in NIMoEsh protected cultures. $\boldsymbol{E}$, Dose-response of NMDA-challenged neuronal cultures to NIMoE with indicated dosages, as assessed by LDH assays. $\boldsymbol{F}$, NIMoE protects neuronal cultures against a range of NMDA concentrations as indicated. $t$ test. ${ }^{*} p<0.05$. Error bars show means \pm SEM.

By studying activation mechanisms of the zD17-JNK module in excitotoxicity, we provide some clues on this issue. Although zD17-promoted activation of JNK is independent of PAT activity, substrate binding to $\mathrm{zD} 17$, which is dependent on the palmitoylation status of the substrates (Huang et al., 2009), regulates the $\mathrm{zD} 17-\mathrm{JNK}$ module and activation of JNK. This suggests that the different functions of zD17 are systematically connected and may directly or indirectly contribute to the pathogenesis of neurological diseases (Singaraja et al., 2002; Yanai et al., 2006; Goytain et al., 2008). Altered palmitoylation and interaction profiles of zD17 substrates, as well as activation of JNK pathways, have been noted in several neurological diseases (Kornau et al., 
A

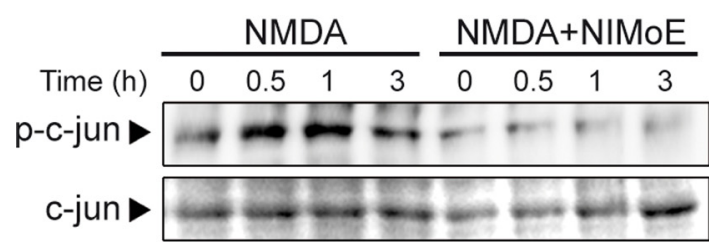

B

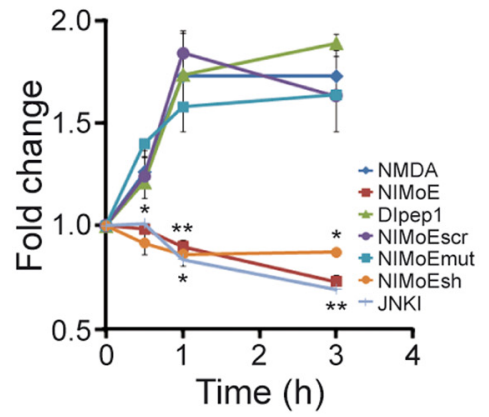

F

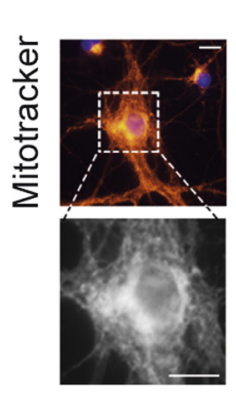

D
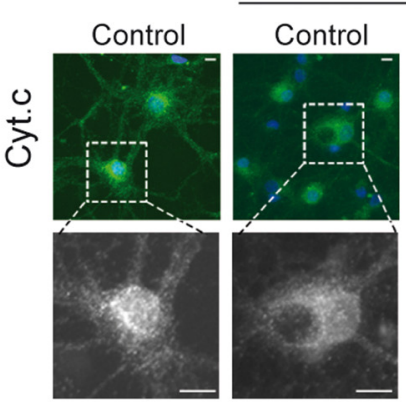

C

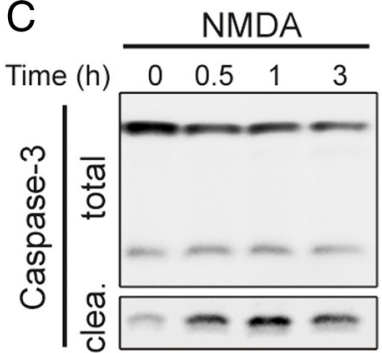

$\mathrm{NMDA}+\mathrm{NIMOE}$

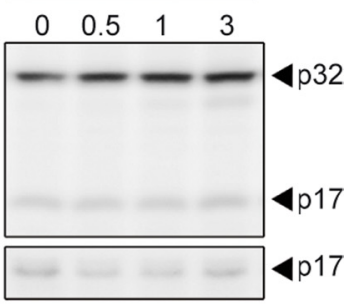

E

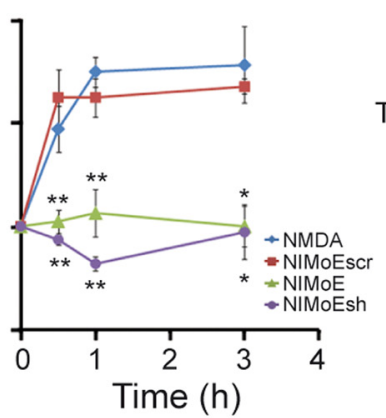

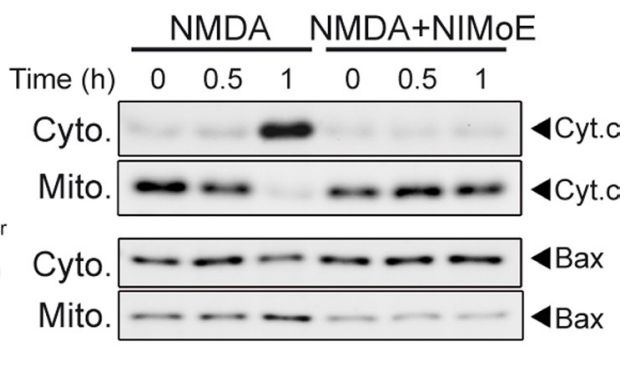

NMDA
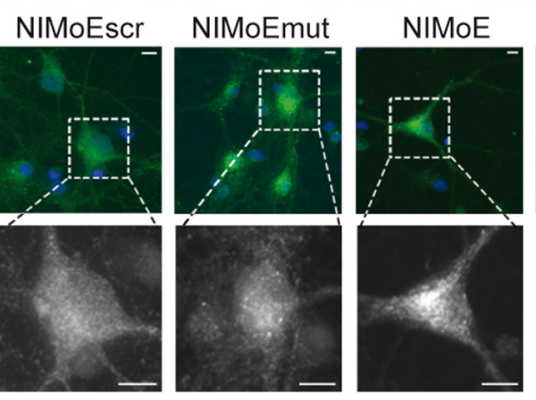

NIMoEsh
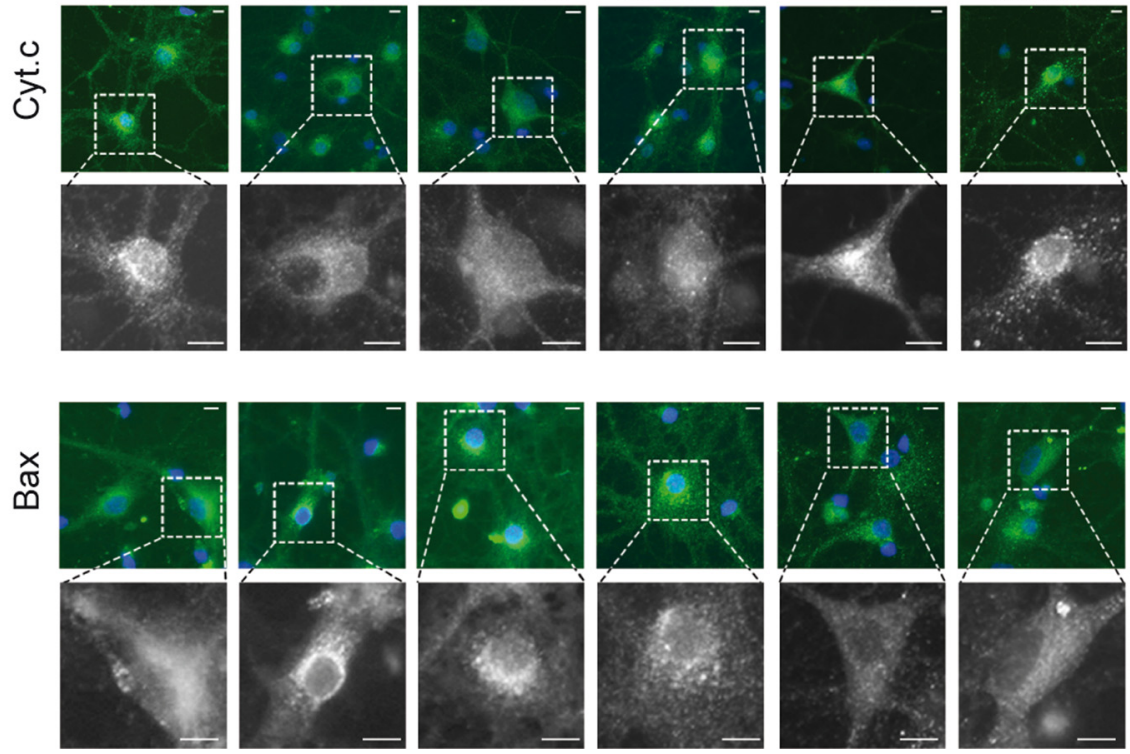

Figure 8. Inhibition of downstream neuronal cell death pathways. A, NMDA-induced c-Jun phosphorylation is blocked by NIMoE treatment. Phosphorylation of c-Jun was quantified in B. $C$ NMDA-induced caspase-3 cleavage is blocked by NIMoE. Caspase-3 was blotted with an antibody either recognizing both p32 and p17 (total) or only the p17 cleaved form of caspase-3 (clea.). The p17 form is quantified in $\boldsymbol{D}$. One-way ANOVA test. ${ }^{*} p<0.05$ and ${ }^{* *} p<0.01$, respectively. Error bars show means \pm SEM. $\boldsymbol{E}$, Translocation of Cyt c and Bax are blocked by NIMoE. Cytosolic (Cyto.) and mitochondria (Mito.) fractions were isolated as mentioned in Materials and Methods, followed by detection of Cyt c and Bax with SDS-PAGE. $\boldsymbol{F}$, The effects of the peptides on translocation of Bax and Cyt c. Mitochondria were labeled with MitoTracker-TR (red). After fixation, Bax and Cyt c were detected with specific antibodies (green). Scale bar, $10 \mu \mathrm{m}$.

1995; El-Husseini et al., 2002; Yanai et al., 2006). Additional investigations on how zD17 relates to its substrates to regulate JNK activation in different pathological conditions may lead to new approaches to treat neurological diseases.

Similar to zD17, several JNK-interacting proteins, or JNK scaffold proteins, have been suggested to play a role in neurological diseases (Morrison and Davis, 2003; Weston and Davis, 2007). Using these proteins as therapeutic targets has attractive advantages because it may inhibit JNK activation in a scenarioselective manner and thus reduce side effects caused by global
JNK inhibition. Despite the identification of JNK-binding motifs from several JNK scaffold proteins (Morrison and Davis, 2003), successful manipulation of their interactions with JNK and blockade of pathological activation of JNK has not been achieved. In the case of the newly developed peptide inhibitor JNKI, the JNK-binding motif on JIP, JNK-interacting protein, was designed to block the JIP-JNK interaction (Borsello et al., 2003). However, it was found to be more effective in blocking the interaction of JNK with its substrate c-Jun and showed no effect on JNK activation (Borsello et al., 2003). This may be because the 
A

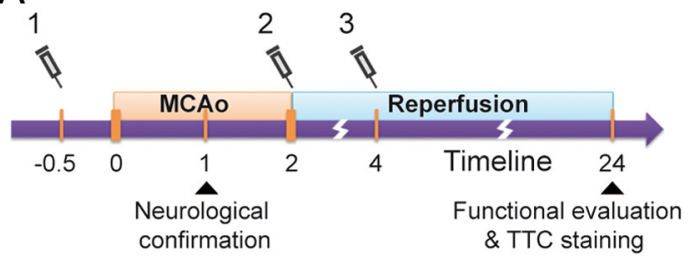

B

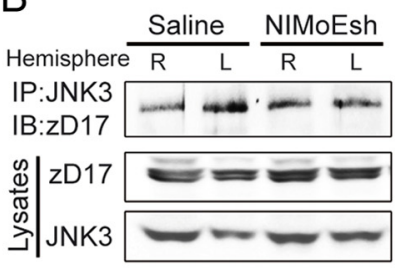

C

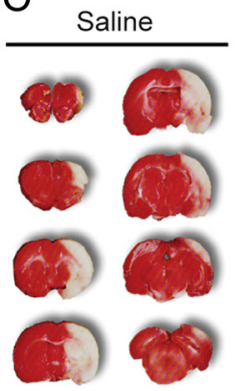

E

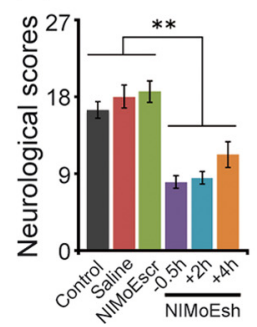

NIMoEsh

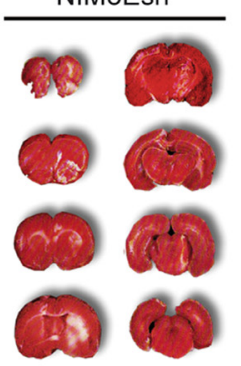

$\mathrm{F}$

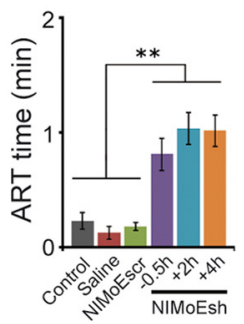

$\mathrm{D}$

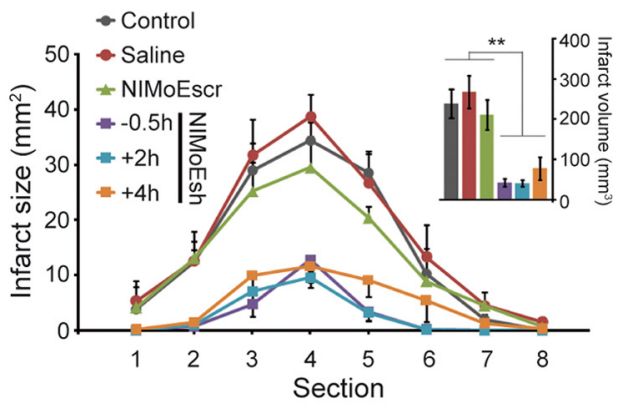

G

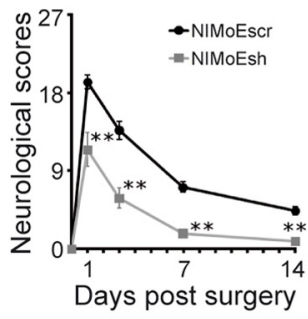

$\mathrm{H}$

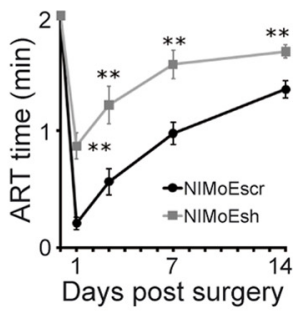

Figure 9. Blocking the JNK-ZD17 interaction with NIMoEsh protects from ischemic brain injury in rats. $\boldsymbol{A}$, The diagram shows the procedures for MCAo experiments. SD rats received sham (control), saline, NIMoEscr, or NIMoEsh by a single intravenous injection at the indicated time before or after the onset of ischemia. $\boldsymbol{B}$, Ischemia induces an enhancement of the zD17-JNK3 interaction, which is blocked by NIMoEsh. Peptide administration 30 min before the ischemic insult significantly blocked the enhancement of interaction induced by MCAo in the injured ipsilateral hemisphere (fold change normalized to the contralateral side; MCAo, $1.64 \pm 0.13, p<0.05 ;$ MCAo + NIMoEsh, $0.85 \pm 0.15, p=0.33) . t$ test. C, Representative TTC (2,3,7triphenyltetrazolium chloride)-stained brain sections from saline- and NIMoEsh-treated (30 min before MCAo onset) groups. $\boldsymbol{D}_{\text {, }}$ Quantification of total infarct size and volume (inset bar chart). NIMoEsh treatments $(-0.5 \mathrm{~h}, 43.43 \pm 9.82, n=7 ;+2 \mathrm{~h}$, $41.95 \pm 8.22, n=10 ;+4 h, 78.74 \pm 28.50, n=6 ; F=20.71, p<0.01$ compared with control groups) dramatically reduced total infarct volume, whereas saline or NIMoEscr had little effect (control, $240.70 \pm 35.67, n=9$; saline, $268.89 \pm 40.47, n=4$; NIMoEscr, $212.60 \pm 37.6, n=5 ; F=0.38, p=0.69$ ). One-way ANOVA test. $\boldsymbol{E}$, Neurological scores indicate an improved behavioral outcome in ischemic rats treated with NIMoEsh. Neurological functions were examined $22 \mathrm{~h}$ after the onset of MCA0. Saline- or NIMoEscr-treated groups showed similar deficits (control, $16.43 \pm 0.95, n=7$; saline, $18.00 \pm 1.35, n=4$; NIMoEscr, $18.60 \pm 1.24, n=5 ; F=1.65, p=0.23)$. Administration of NIMoEsh significantly reduced neurological scores $(-0.5 \mathrm{~h}, 8.00 \pm$ $0.72, n=7 ;+2 \mathrm{~h}, 8.50 \pm 0.75, n=10 ;+4 \mathrm{~h}, 11.20 \pm 1.49, n=6 ; F=21.98, p<0.01$ compared with control groups). One-way ANOVA test. $\boldsymbol{F}$, NIMoE treatment improves somatosensory functions as assessed with the ART. MCAo eliminates responses on the contralateral side in control, saline-treated, and NIMoEscr-treated animals (control, $13.57 \pm 4.30 \mathrm{~s}, n=7$; saline, $7.42 \pm$ $3.21 \mathrm{~s}, n=4 ; \mathrm{NIMoEscr}, 10.80 \pm 2.06 \mathrm{~s}, n=5 ; F=0.64, p=0.54$ ). Pretreatment or posttreatment infusion of NIMoE led to an improvement in somatosensory functions $(-0.5 \mathrm{~h}, 48.62 \pm 8.24 \mathrm{~s}, n=7 ;+2 \mathrm{~h}, 62.1 \pm 8.60 \mathrm{~s}, n=10 ;+4 \mathrm{~h}, 60.83 \pm 8.13 \mathrm{~s}$, $n=6 ; F=11.28, p<0.01)$. One-way ANOVA test. $\mathbf{G}, \boldsymbol{H}$, Functional tests show improved behavioral outcomes in NIMoEsh-treated animals after MCAo injury. NIMoEscr or NIMoEsh were given $4 \mathrm{~h}$ after the MCAo onset. Neurological scores (NIMoEscr, $n=8$; day 1, $19.25 \pm 0.80$; day 3, $13.63 \pm 1.03$; day 7, $7.13 \pm 0.58$; day 14, $4.38 \pm 0.50$; NIMoEsh, $n=7$; day 1, $11.43 \pm 1.96$; day 3, $5.86 \pm 1.14 ;$ day 7, $1.71 \pm 0.47$; day 14,0.85 \pm 0.34 ) and ART (NIMoEscr, $n=8 ;$ day 1, $12.63 \pm 3.14$; day 3, $34.13 \pm 6.88$; day $7,59.13 \pm 5.67$; day $14,81.88 \pm 4.44 ;$ NIMoEsh, $n=7$; day 1, $52.57 \pm 6.82 ;$ day 3, $73.86 \pm 9.63 ;$ day $7,94.86 \pm 7.33 ;$ day 14 , $101.43 \pm 3.35)$. $t$ test. ${ }^{* *} p<0.01$. Error bars show means \pm SEM. IP, Immunoprecipitation; IB, immunoblot.

JNK-binding motif on the scaffold protein JIP shares the same sequence with the JNK substrate c-Jun (Borsello et al., 2003). In contrast, the JNK-binding motifs that we have identified here appear to be unique to $\mathrm{zD} 17$. As we demonstrate with the peptide NIMoE, this provides an opportunity to manipulate JNK activation selectively under stress conditions.

Another challenge for JNK inhibition as a neuroprotective therapy for ischemic stroke or other neurological diseases is JNK isoform selectivity (Bogoyevitch, 2006). Although the advantage of isoform-selective JNK inhibition has long been appreciated, the application of small-molecule inhibitors with this feature in treating ischemic stroke has not been reported (Resnick and Fennell, 2004; Bogoyevitch, 2006). The development of small molecules with isoform selectivity has proven challenging because JNK isoforms are highly conserved at the ATPbinding sites. An alternative strategy is to take advantage of JNK-interacting proteins, some of which have been shown to have selective binding preferences to JNK isoforms (Kelkar et al., 2000; McDonald et al., 2000). Using these proteins as targets, however, no JNK isoform-selective inhibition has been successfully developed as a therapeutic strategy in neurological diseases.

zD17 shows several attractive attributes to make it an appealing target. First, the $\mathrm{zD} 17-\mathrm{JNK}$ interaction responds to diverse neuronal stresses, including excitotoxicity and inflammation (Fig. 3), implying that the zD17-JNK signaling module may contribute to JNK activation at different stages in the ischemic process. In fact, an increase of the zD17-JNK interaction is detected up to at least $6 \mathrm{~h}$ after ischemia onset (Fig. 9), when the stage of excitotoxicity is already believed to be waning (Lo, 2009). This suggests a broad time window for therapeutic intervention. Second, the activation mechanism of the zD17-JNK signaling module suggests a central position of $\mathrm{zD} 17$ in sensing upstream stress signals and generation of outputs to JNK pathways. As we shown with PSD-95, substrate binding represents one candidate mechanism that links excitotoxicity to JNK activation via $\mathrm{zD} 17$ (Fig. 3). Other mechanisms may also contribute to the regulation of $\mathrm{zD} 17$ mediated JNK activation. It is interesting that another PAT, zD13, is also able to interact with JNK, although it is not efficient as zD17 in promoting JNK phosphorylation (Fig. $1 F$ ). Additional studies on the role of zD13 and other potential regulators on JNK activation would bring more insights on the regulatory mechanism of the $\mathrm{zD} 17-\mathrm{JNK}$ signaling module. Third, the stress-induced enhancement of the $\mathrm{zD} 17-\mathrm{JNK}$ module shows a marked preference for JNK isoforms 2 and 3. Fourth, activation of JNK2/3 and downstream cell death pathways are primarily mediated by the zD17-JNK module, and finally, a specific JNK-binding motif on zD17 (motif E) is selectively recruited to enhance the $\mathrm{zD} 17-\mathrm{JNK}$ interaction under stress conditions.

By targeting zD17 with peptides derived from motif $\mathrm{E}$ (NIMoE/NIMoEsh), we show the following: (1) JNK can be inhibited with selectivity to isoforms JNK2/3; (2) inhibition of JNK 
is only effective when the zD17-JNK interaction is enhanced under stress conditions; (3) normal functions of JNK pathways are preserved, as we assessed with studies of axonal growth; (4) excitotoxicity-induced neuronal cell death is markedly prevented in cultured neurons; and (5) brain damage and behavioral deficits in rats subjected to ischemia are substantially reduced over a broad time window. These studies establish the $\mathrm{zD} 17-J N K 3$ interaction as a candidate target with reduced side effects for ischemic stroke. However, several additional steps, such as recruitment of additional stroke models and different animal species, are still required to more fully validate the therapeutic potential of this new target (Stroke Therapy Academic Industry Roundtable, 1999).
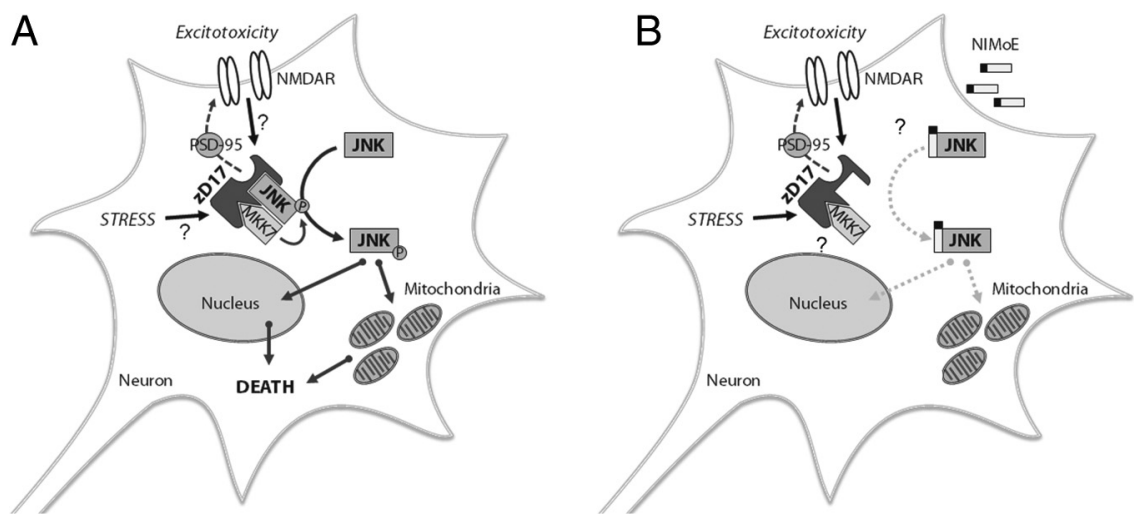

Figure 10. Potential mechanisms of ZD17-mediated JNK activation $(\boldsymbol{A})$ and the intervention for neuroprotection $(\boldsymbol{B}) . \boldsymbol{A}$, In response to excitotoxic and other stresses, ZD17 interacts with JNK and recruits MKK7 to activate JNK (primarily JNK isoforms 2 and 3) by phosphorylation. Activated JNK turns on downstream cell death pathways in the nucleus and mitochondria. Excitotoxicity promotes the ZD17-JNK interaction partially through the dissociation of ZD17 from PSD-95 that competes with JNK in binding ZD17. Other pathways that may contribute to the regulation of the JNK-ZD17-MKK7 module remain to be elucidated. $\boldsymbol{B}$, The Tat-fused peptides (NIMoE/NIMoEsh) derived from the JNK-binding motif on zD17 blocks the excitotoxic-induced interaction of ZD17 and JNK and thus prevents JNK activation and neuronal cell death.

\section{References}

Aarts M, Liu Y, Liu L, Besshoh S, Arundine M, Gurd JW, Wang YT, Salter MW, Tymianski M (2002) Treatment of ischemic brain damage by perturbing NMDA receptor-PSD-95 protein interactions. Science 298:846-850.

Bederson JB, Pitts LH, Tsuji M, Nishimura MC, Davis RL, Bartkowski H (1986) Rat middle cerebral artery occlusion: evaluation of the model and development of a neurologic examination. Stroke 17:472-476.

Belayev L, Alonso OF, Busto R, Zhao W, Ginsberg MD (1996) Middle cerebral artery occlusion in the rat by intraluminal suture. Neurological and pathological evaluation of an improved model. Stroke 27:1616-1622; discussion 1623.

Bijlmakers MJ, Marsh M (2003) The on-off story of protein palmitoylation. Trends Cell Biol 13:32-42.

Bogoyevitch MA (2006) The isoform-specific functions of the c-Jun $\mathrm{N}$-terminal kinases (JNKs): differences revealed by gene targeting. Bioessays 28:923-934.

Borsello T, Forloni G (2007) JNK signalling: a possible target to prevent neurodegeneration. Curr Pharm Des 13:1875-1886.

Borsello T, Clarke PG, Hirt L, Vercelli A, Repici M, Schorderet DF, Bogousslavsky J, Bonny C (2003) A peptide inhibitor of c-Jun N-terminal kinase protects against excitotoxicity and cerebral ischemia. Nat Med 9:1180-1186.

Bossy-Wetzel E, Schwarzenbacher R, Lipton SA (2004) Molecular pathways to neurodegeneration. Nat Med [Suppl]10:S2-S9.

Bouet V, Boulouard M, Toutain J, Divoux D, Bernaudin M, Schumann-Bard P, Freret T (2009) The adhesive removal test: a sensitive method to assess sensorimotor deficits in mice. Nat Protoc 4:1560-1564.

Bredesen DE, Rao RV, Mehlen P (2006) Cell death in the nervous system. Nature 443:796-802.

Centeno C, Repici M, Chatton JY, Riederer BM, Bonny C, Nicod P, Price M, Clarke PG, Papa S, Franzoso G, Borsello T (2007) Role of the JNK pathway in NMDA-mediated excitotoxicity of cortical neurons. Cell Death Differ 14:240-253.

Chang L, Jones Y, Ellisman MH, Goldstein LS, Karin M (2003) JNK1 is required for maintenance of neuronal microtubules and controls phosphorylation of microtubule-associated proteins. Dev Cell 4:521-533.

Coffey ET, Smiciene G, Hongisto V, Cao J, Brecht S, Herdegen T, Courtney MJ (2002) c-Jun N-terminal protein kinase (JNK) $2 / 3$ is specifically activated by stress, mediating c-Jun activation, in the presence of constitutive JNK1 activity in cerebellar neurons. J Neurosci 22:4335-4345.

Donnan GA, Fisher M, Macleod M, Davis SM (2008) Stroke. Lancet 371:1612-1623.

el-Husseini Ael-D, Bredt DS (2002) Protein palmitoylation: a regulator of neuronal development and function. Nat Rev Neurosci 3:791-802.

El-Husseini Ael-D, Schnell E, Dakoji S, Sweeney N, Zhou Q, Prange O, Gauthier-Campbell C, Aguilera-Moreno A, Nicoll RA, Bredt DS (2002)
Synaptic strength regulated by palmitate cycling on PSD-95. Cell 108:849-863.

Fisher M, Ratan R (2003) New perspectives on developing acute stroke therapy. Ann Neurol 53:10-20.

Goytain A, Hines RM, Quamme GA (2008) Huntingtin-interacting proteins, HIP14 and HIP14L, mediate dual functions, palmitoyl acyltransferase and $\mathrm{Mg}^{2+}$ transport. J Biol Chem 283:33365-33374.

Harada T, Matsuzaki O, Hayashi H, Sugano S, Matsuda A, Nishida E (2003) AKRL1 and AKRL2 activate the JNK pathway. Genes Cells 8:493-500.

Huang K, Yanai A, Kang R, Arstikaitis P, Singaraja RR, Metzler M, Mullard A, Haigh B, Gauthier-Campbell C, Gutekunst CA, Hayden MR, El-Husseini A (2004) Huntingtin-interacting protein HIP14 is a palmitoyl transferase involved in palmitoylation and trafficking of multiple neuronal proteins. Neuron 44:977-986.

Huang K, Sanders S, Singaraja R, Orban P, Cijsouw T, Arstikaitis P, Yanai A, Hayden MR, El-Husseini A (2009) Neuronal palmitoyl acyl transferases exhibit distinct substrate specificity. FASEB J 23:2605-2615.

Hunot S, Vila M, Teismann P, Davis RJ, Hirsch EC, Przedborski S, Rakic P, Flavell RA (2004) JNK-mediated induction of cyclooxygenase 2 is required for neurodegeneration in a mouse model of Parkinson's disease. Proc Natl Acad Sci USA 101:665-670.

Kang R, Wan J, Arstikaitis P, Takahashi H, Huang K, Bailey AO, Thompson JX, Roth AF, Drisdel RC, Mastro R, Green WN, Yates JR 3rd, Davis NG, El-Husseini A (2008) Neural palmitoyl-proteomics reveals dynamic synaptic palmitoylation. Nature 456:904-909.

Kelkar N, Gupta S, Dickens M, Davis RJ (2000) Interaction of a mitogenactivated protein kinase signaling module with the neuronal protein JIP3. Mol Cell Biol 20:1030-1043.

Khatlani TS, Wislez M, Sun M, Srinivas H, Iwanaga K, Ma L, Hanna AE, Liu D, Girard L, Kim YH, Pollack JR, Minna JD, Wistuba II, Kurie JM (2007) c-Jun N-terminal kinase is activated in non-small-cell lung cancer and promotes neoplastic transformation in human bronchial epithelial cells. Oncogene 26:2658-2666.

Kornau HC, Schenker LT, Kennedy MB, Seeburg PH (1995) Domain interaction between NMDA receptor subunits and the postsynaptic density protein PSD-95. Science 269:1737-1740.

Kuan CY, Burke RE (2005) Targeting the JNK signaling pathway for stroke and Parkinson's diseases therapy. Curr Drug Targets CNS Neurol Disord 4:63-67.

Lo EH (2009) T time in the brain. Nat Med 15:844-846.

Longa EZ, Weinstein PR, Carlson S, Cummins R (1989) Reversible middle cerebral artery occlusion without craniectomy in rats. Stroke 20:84-91.

McDonald PH, Chow CW, Miller WE, Laporte SA, Field ME, Lin FT, Davis RJ, Lefkowitz RJ (2000) Beta-arrestin 2: a receptor-regulated MAPK scaffold for the activation of JNK3. Science 290:1574-1577.

Merritt SE, Mata M, Nihalani D, Zhu C, Hu X, Holzman LB (1999) The 
mixed lineage kinase DLK utilizes MKK7 and not MKK4 as substrate. J Biol Chem 274:10195-10202.

Morrison DK, Davis RJ (2003) Regulation of MAP kinase signaling modules by scaffold proteins in mammals. Annu Rev Cell Dev Biol 19:91-118.

Nagel S, Papadakis M, Chen R, Hoyte LC, Brooks KJ, Gallichan D, Sibson NR, Pugh C, Buchan AM (2011) Neuroprotection by dimethyloxalylglycine following permanent and transient focal cerebral ischemia in rats. J Cereb Blood Flow Metab 31:132-143.

Oliva AA Jr, Atkins CM, Copenagle L, Banker GA (2006) Activated c-Jun $\mathrm{N}$-terminal kinase is required for axon formation. J Neurosci 26: 9462-9470.

Resnick L, Fennell M (2004) Targeting JNK3 for the treatment of neurodegenerative disorders. Drug Discov Today 9:932-939.

Schwarze SR, Ho A, Vocero-Akbani A, Dowdy SF (1999) In vivo protein transduction: delivery of a biologically active protein into the mouse. Science 285:1569-1572.

Singaraja RR, Hadano S, Metzler M, Givan S, Wellington CL, Warby S, Yanai A, Gutekunst CA, Leavitt BR, Yi H, Fichter K, Gan L, McCutcheon K, Chopra V, Michel J, Hersch SM, Ikeda JE, Hayden MR (2002) HIP14, a novel ankyrin domain-containing protein, links huntingtin to intracellular trafficking and endocytosis. Hum Mol Genet 11:2815-2828.

Song X, Raman D, Gurevich EV, Vishnivetskiy SA, Gurevich VV (2006) Visual and both non-visual arrestins in their "inactive" conformation bind JNK3 and Mdm2 and relocalize them from the nucleus to the cytoplasm. J Biol Chem 281:21491-21499.
Stroke Therapy Academic Industry Roundtable (STAIR) (1999) Recommendations for standards regarding preclinical neuroprotective and restorative drug development. Stroke 30:2752-2758.

Tsuruta F, Sunayama J, Mori Y, Hattori S, Shimizu S, Tsujimoto Y, Yoshioka K, Masuyama N, Gotoh Y (2004) JNK promotes Bax translocation to mitochondria through phosphorylation of 14-3-3 proteins. EMBO J 23:1889-1899.

Watanabe T, Okuda Y, Nonoguchi N, Zhao MZ, Kajimoto Y, Furutama D, Yukawa H, Shibata MA, Otsuki Y, Kuroiwa T, Miyatake S (2004) Postischemic intraventricular administration of FGF-2 expressing adenoviral vectors improves neurologic outcome and reduces infarct volume after transient focal cerebral ischemia in rats. J Cereb Blood Flow Metab 24:1205-1213.

Weston CR, Davis RJ (2007) The JNK signal transduction pathway. Curr Opin Cell Biol 19:142-149.

WHO (2007) The world health report: report of the Director-General. Geneva: World Health Organization.

Yanai A, Huang K, Kang R, Singaraja RR, Arstikaitis P, Gan L, Orban PC, Mullard A, Cowan CM, Raymond LA, Drisdel RC, Green WN, Ravikumar B, Rubinsztein DC, El-Husseini A, Hayden MR (2006) Palmitoylation of huntingtin by HIP14 is essential for its trafficking and function. Nat Neurosci 9:824-831.

Yang G, Xiong W, Kojic L, Cynader MS (2009) Subunit-selective palmitoylation regulates the intracellular trafficking of AMPA receptor. Eur J Neurosci 30:35-46. 